\title{
Evaluation and error analysis: Kalman gain regularization versus covariance regularization
}

\author{
Yanfen Zhang • Dean S. Oliver
}

Received: 21 April 2010 / Accepted: 22 November 2010 / Published online: 6 January 2011

(C) The Author(s) 2011. This article is published with open access at Springerlink.com

\begin{abstract}
Ensemble size is critical to the efficiency and performance of the ensemble Kalman filter, but when the ensemble size is small, the Kalman gain generally cannot be well estimated. To reduce the negative effect of spurious correlations, a regularization process applied on either the covariance or the Kalman gain seems to be necessary. In this paper, we evaluate and compare the estimation errors when two regularization methods including the distance-dependent localization and the bootstrap-based screening are applied on the covariance and on the Kalman gain. The investigations were carried out through two examples: 1D linear problem without dynamics but for which the true Kalman gain can be computed and a 2D highly nonlinear reservoir fluid flow problem. The investigation resulted in three primary conclusions. First, if localizations of two covariance matrices are not consistent, the estimate of the Kalman gain will generally be poor at the observation location. The consistency condition can be difficult to apply for nonlocal observations. Second, the estimate of the Kalman gain that results from covariance regularization is generally subject to greater errors than the estimate of the Kalman gain that results from Kalman gain regularization. Third, in terms of removing spurious correlations in the estimation of spatially correlated variables, the performance of screen-
\end{abstract}

\footnotetext{
Y. Zhang $(\bowtie)$

Chevron Energy Technology Company, 1500 Louisiana St., Houston, TX 77002, USA

e-mail: Yanfen.Zhang@chevron.com,z6y7f8@gmail.com

D. S. Oliver

Uni Research, Centre for Integrated Petroleum Research,

P.O. Box 7800, 5007 Bergen, Norway

e-mail: dean.oliver@uni.no
}

ing Kalman gain is comparable as the performance of localization methods (applied on either covariance or Kalman gain), but screening Kalman gain outperforms the localization methods in terms of generality for application, as the screening method can be used for estimating both spatially correlated and uncorrelated variables, and moreover, no assumption about the prior covariance is required for the screening method.

Keywords Kalman gain regularization • Covariance regularization - Distance-dependent localization - Bootstrap-based screening • Ensemble Kalman filter $\cdot$ History matching

\section{Introduction}

In petroleum engineering, the ensemble Kalman filter (EnKF) [7] is frequently used for estimating large numbers $\left(10^{5}-10^{6}\right)$ of reservoir model parameters and dynamic state variables by assimilating the historical production data $[8,11,13,18,20]$. Although the update step in EnKF assumes linearity in the relationship of observations to model and state variables, the forecast step in the EnKF is done by running a nonlinear numerical reservoir simulator, which is usually more expensive than the matrix computations in the update (or analysis) step. For computational efficiency, it is always desirable to use a small ensemble size. One problem that arises as a result of a small ensemble size is that of spurious correlations, which can lead to unrealistic updates to the model parameters and state variables. The cumulative effect of unrealistic updates is the loss of the ensemble variability and final breakdown of EnKF [17]. 
For reducing the negative effect of spurious correlations and improving the effective rank, it is necessary to have a denoising process applied on either the covariance or the Kalman gain. The most common method for reducing the effect is distance-dependent covariance localization. The concept of localization in the EnKF framework was first introduced by Houtekamer and Mitchell [14]. Since then, the localization method has evolved from a distance cutoff approach to a tapering form $[12,15]$. The main parameter in a distance-based tapering function is the range or correlation length, which defines the domain of true correlations [16]. An optimal value for range of the taper function, however, is not easily determined [10,17]. The concept of localization was originally applied to the covariance matrix. Improved results, however, were also obtained by applying localization on the Kalman gain [1, 2, 5, 20]. In spite of the widespread applications of these two ways of using localization, little in the literature addresses the difference between these two ways of applying localization. This paper presents a comparison study between the covariance localization and the Kalman gain localization.

Distance-dependent localization is an effective method, but there are some challenges associated with this method. Chen and Oliver [4] applied distancebased covariance localization in sequential data assimilation for multiphase flow. The authors showed that the appropriate localization pattern or tapering function may vary with different types of data and model parameters; moreover, the optimal localization depends on the history of previous data assimilation. It is clear that many factors must be considered when applying distance-based localization methods. In addition, distance-dependent covariance localization is only appropriate for spatially correlated variables, and it is not suitable for "localizing" global reservoir variables such as fluid contacts and relative permeability parameters. Methods without the assumption of distance dependence are needed for dealing with the sampling error caused by a small ensemble size. Motivated by the work of Anderson [2], Zhang and Oliver [21] developed a statistics-based screening algorithm, in which bootstrap resampling is used to assess the confidence level of each element from the Kalman gain matrix and to filter out the unrealistic correlations from the Kalman gain. The bootstrap-based screening method was demonstrated to be effective at eliminating unrealistic correlations and easy to implement. In this study, we present a further investigation on the bootstrap-based screening methods applied on both the Kalman gain and the covariance. The investigations are carried out through two examples: a 1D linear problem for which the exact solution can be computed and a 2D highly nonlinear reservoir fluid flow problem. Consistency conditions and error evolution in the screening and localization are analyzed. The applicability of both methods are discussed.

\section{The ensemble Kalman filter}

The ensemble Kalman filter is a reduced-rank sequential data assimilation method. In the standard implementation of the ensemble Kalman filter, the probability density function is approximated by an ensemble of $N_{e}$ state vectors,

$Y=\left[y_{1}, y_{2}, \ldots, y_{N_{e}}\right]$

where each state vector consists of the dynamic state variables $v_{i}$ and/or static model parameters $m_{i}$. Dynamic state variables are function of model parameters and change with time. For petroleum inverse problem, we usually include both the static model parameters and the dynamic state variables in the state vector

$y_{i}=\left[\begin{array}{c}m_{i} \\ v_{i}\end{array}\right], \quad i=1,2, \ldots, N_{e}$.

The ensemble Kalman filter consists of two recursive steps. First is the forecast step in which the model parameters $m_{i}$ remain the same,

$m_{t+1, i}=m_{t, i}$,

while the dynamic model variables $v_{i}$ are evolved from time $t$ to time $t+1$,

$v_{t+1, i}=f\left(y_{t, i}\right)$.

The predicted data for the $i$-th ensemble member at time $t+1, d_{t+1, i}^{f}$, are computed from the model and state variables by running a forward model. In petroleum application, the forward model is a numerical reservoir simulator,

$d_{t+1, i}^{f}=g\left(y_{t+1, i}\right) \quad i=1,2, \ldots, N_{e}$

where $g(\cdot)$ denotes the observation operator.

In the analysis or updating step, both the forecast model parameters and state variables are updated,

$Y^{a}=Y^{f}+K_{e}\left(d^{\mathrm{obs}}-d^{f}\right)$.

In this expression, the subscript of time index is neglected, $d^{\text {obs }}$ are perturbed observations obtained by adding zero-mean noise with covariance $C_{D}$ to the 
actual measurement values, and $K_{e}$ is the Kalman gain that is computed from the forecast ensemble $Y^{f}$ and predicted data $d^{f}$, using the expression

$K_{e}=C_{y d}^{f}\left(C_{d d}^{f}+C_{D}\right)^{-1}$

where $C_{y d}^{f}$ is the covariance between the variables in the state vector and predicted data and $C_{d d}^{f}$ is the covariance between different predicted data. $C_{y d}^{f}$ and $C_{d d}^{f}$ are estimated from the ensemble. When the ensemble size is limited, we must deal with the issues of rank deficiency and spurious correlations present in the covariances and the corresponding Kalman gain. In the following two sections, we present two denoising algorithms: distance-dependent localization and bootstrapbased screening.

\section{The distance-dependent localization}

Distance-dependent localization is the most common method for eliminating spurious correlations. Generally, localization is applied on the covariances by taking the Schur product of covariances with the localization coefficients.

$K_{e}^{\mathrm{LC}}=C_{y d}^{f} \circ \beta_{y d}\left(C_{d d}^{f} \circ \beta_{d d}+C_{D}\right)^{-1}$

where superscript LC stands for localizing covariance and $\circ$ denotes a Schur or Hadamard product. $C_{y d}^{f}$ and $C_{d d}^{f}$ are two related components of the Kalman gain. To illustrate the relationship, the two covariances are rewritten in terms of sensitivity matrix of simulated data with respect to state vector, $G=\left(\nabla g^{\mathrm{T}}\right)^{\mathrm{T}}$,

$$
\begin{aligned}
C_{y d}^{f} & =E\left[(y-\bar{y})(g(y)-\bar{g}(y))^{\mathrm{T}}\right] \\
& \approx E\left[(y-\bar{y})(y-\bar{y})^{\mathrm{T}} G^{\mathrm{T}}\right] \\
& \approx C_{y y}^{f} G^{\mathrm{T}} \\
C_{d d}^{f} & =E\left[(g(y)-\bar{g}(y))(g(y)-\bar{g}(y))^{\mathrm{T}}\right] \\
& \approx E\left[G(y-\bar{y})(y-\bar{y})^{\mathrm{T}} G^{\mathrm{T}}\right] \\
& \approx G C_{y y}^{f} G^{\mathrm{T}} .
\end{aligned}
$$

Thus, the relationship between the two covariance matrices is $C_{d d}^{f}=G C_{y d}^{f}$ or $C_{d d}^{f}=\left(C_{y d}^{f}\right)^{\mathrm{T}}\left(C_{y y}^{f}\right)^{-1} C_{y d}^{f}$. Because the two matrices $C_{d d}^{f}$ and $C_{y d}^{f}$ are not independent and the relationship between them must be satisfied after localization or screening, there is a consistency re- quirement for applying covariance localization or other screening algorithms on covariances. If we assume that the same consistency condition applies to the localized covariance, then

$C_{d d}^{f} \circ \beta_{d d}=G\left(C_{y d}^{f} \circ \beta_{y d}\right)$

or

$C_{d d}^{f} \circ \beta_{d d}=\left(C_{y d}^{f} \circ \beta_{y d}\right)^{\mathrm{T}}\left(C_{y y}^{f} \circ \beta_{y y}\right)^{-1}\left(C_{y d}^{f} \circ \beta_{y d}\right)$.

For cases in which $G$ can be solved efficiently and the cost of computing the full covariance matrix $C_{y y}^{f}$ can also be afforded, there is no need to worry about consistency issue because the localized $C_{y d}^{f}$ and localized $C_{d d}^{f}$ can be obtained using Eqs. 3 and 4 with the localized full covariance $C_{y y}^{f} \circ \beta_{y y}$. For most practical applications, however, the problem is nonlinear and high dimensional, $G$ cannot be computed efficiently, and we cannot afford to calculate the full covariance. Thus, our starting point is the covariances $C_{y d}^{f}$ and $C_{d d}^{f}$, and the problem is to reduce spurious correlations in these two matrices through construction of taper matrices $\beta_{y d}$ and $\beta_{d d}$ that satisfy the consistency conditions (Eq. 5 or Eq. 6). $\beta_{y d}$ always can be defined according to the prior model, but it is not trivial to build $\beta_{d d}$ that is consistent with $\beta_{y d}$ when the observations are non-local. An observation is termed "non-local" if it is sensitive to state or model variables over an extended region. A simple example of a non-local observation in petroleum engineering is an observation of water production rate at a producing well, as it is sensitive to the porosity in the region between the injector and the producer. Examples from meteorology and possible methods for handling assimilation of non-local data can be found in Fertig et al. [9]. So far, no general methods for defining consistent $\beta_{y d}$ and $\beta_{d d}$ are available.

Instead of applying localization to the covariance matrices, an alternative is to apply the localization directly on the Kalman gain by performing a Schur product with $\beta_{y d}[3,5,20]$,

$K_{e}^{\mathrm{LK}}=\beta_{y d} \circ K_{e}$

where superscript LK stands for localizing Kalman gain. Kalman gain localization avoids the inconsistency issue but presents other difficulties as discussed in Section 1. Thus, in the next section, a screening algorithm is discussed as an improved alternative method to localization. 


\section{Bootstrap-based screening}

This screening algorithm is based on a resampling method, bootstrap, which avoids the challenge of evaluating a large number of realizations. No range parameter or other assumption about the prior covariance is required in this algorithm. It can be used for eliminating the spurious correlations for both spatially correlated and uncorrelated variables. The description of the algorithm starts with a brief introduction to bootstrap.

Bootstrap is a nonparametric computer-intensive resampling method for statistical inference. Bootstrapping uses repeated samples from the parent data set to compute the statistics of interest, such as variance, confidence intervals, and bias of an estimator $\hat{\theta}$. In this paper, $\hat{\theta}$ denotes a standard method for estimating a quantity of interest such as the Kalman gain matrix $\left(K_{e}\right)$ or covariance matrices $\left(C_{y d}^{f}\right.$ and $\left.C_{d d}^{f}\right)$. For our problem, the parent data set is the forecast ensemble $\Psi^{f}$ containing $N_{e}$ augmented state vectors. Each augmented state vector is composed of model parameters, state variables, and the corresponding simulated data

$\psi_{i}^{f}=\left[\begin{array}{c}y_{i}^{f} \\ d_{i}^{f}\end{array}\right], \quad i=1,2, \ldots, N_{e}$.

In the bootstrapping framework, a bootstrapped sample of the augmented forecast ensemble, $\Psi^{f^{*}}$, is generated by randomly sampling $N_{e}$ realizations from $\Psi^{f}$ with replacement. Assume that $N_{B}$ bootstrapped samples of the ensemble of augmented state vectors are generated. Because the sampling is done with replacement, the ensembles may contain the same ensemble members multiple times. For each bootstrapped sample, $\Psi^{f^{*}}$, a sample estimate $\hat{\theta}^{*}$ can be computed. Finally, the $N_{B}$ sample estimates $\hat{\theta}^{*}$ form an empirical distribution, which can be used for estimating the variances of the estimator $\hat{\theta}$.

For each element in $\hat{\theta}$, the plug-in estimator of variance is

$\hat{\sigma}_{\theta_{i, j}}^{2}=\frac{\sum_{m=1}^{N_{B}}\left(\hat{\theta}_{i, j, m}^{*}-\bar{\theta}_{i, j}\right)^{2}}{N_{B}}$,

and subsequently, the squared coefficient of variation is defined as the ratio of the variance to the squared mean

$\hat{C}_{v_{i, j}}^{2}=\frac{\hat{\sigma}_{\theta_{i, j}}^{2}}{\bar{\theta}_{i, j}^{2}}$

where the lower and upper limits of subscripts $i$ and $j$ depend on the definition of $\hat{\theta}$. If $\hat{\theta}$ denotes $C_{y d}^{f}$ or $K_{e}$, then $i \in\left[1, N_{y}\right]$ and $j \in\left[1, N_{d}\right]$. If $\hat{\theta}$ denotes $C_{d d}^{f}$, then $i \in\left[1, N_{d}\right]$ and $j \in\left[1, N_{d}\right]$. There is no specific requirement on the value of $N_{B}$. The larger the number of bootstrapped samples, the more reliable the estimate of variation coefficient will be, but $N_{B}=50$ is often enough to give a good estimate of standard error [6].

The purpose of screening is to minimize the difference between the values of the Kalman gain or the covariance estimated from a small ensemble of samples and the true values of those quantities. In order to achieve this, we introduce a screening factor defined as the value of $\alpha$ that minimizes the following objective function

$S(\alpha)=S_{\theta}(\alpha)+S_{\alpha}(\alpha)$

where $S_{\theta}(\alpha)$ is a measure of the difference between the screened estimate of $\theta$ and the true $\theta$,

$S_{\theta}(\alpha)=\frac{1}{2 N_{B}} \sum_{m=1}^{N_{B}}\left\|\left(\alpha \circ \hat{\theta}_{m}^{*}-\bar{\theta}\right) \circ \lambda_{\theta}\right\|_{F}^{2}$,

and where $\lambda_{\theta}$ is a matrix composed of $1 / \hat{\sigma}_{\theta_{i, j}} . S_{\alpha}(\alpha)$ is a regularization term that penalizes large values of $\alpha$. It can be defined as

$S_{\alpha}(\alpha)=\frac{1}{2}\left\|\alpha \circ \lambda_{\alpha}\right\|_{F}^{2}$,

where $\lambda_{\alpha}$ is a matrix containing $1 / \sigma_{\alpha}$ for all entries. If the variables to be updated are spatially correlated and preserving smoothness is desirable, $S_{\alpha}(\alpha)$ could be defined to minimize the magnitude of the derivative of $\alpha$.

After substituting Eqs. 9 and 10 into Eq. 8, the 2nd derivative of $S(\alpha)$ is seen to be positive definite. Thus, the least square solution for $\alpha_{i, j}$ is obtained by differentiating Eq. 8 with respect to $\alpha$ and equating it to zero.

$\alpha_{i, j}=\frac{1}{1+\left(1+1 / \sigma_{\alpha}^{2}\right) \hat{C}_{v_{i, j}}^{2}}$.

The solution is seen to depend on both the squared coefficient of variation, $\hat{C}_{v_{i, j}}^{2}$, and on the value chosen for $\sigma_{\alpha}^{2}$. With a reasonably small value of $\sigma_{\alpha}^{2}$, inclusion of the regularization term $S_{\alpha}(\alpha)$ forces $\alpha_{i, j}$ to be close to 0 . The effectiveness of eliminating spurious correlations increases as the value of $\sigma_{\alpha}^{2}$ is decreased, but, at the same time, the possibility of removing true correlations also increases. There is a tradeoff between the benefit of eliminating spurious correlations and the harm done by removing true correlations that must be balanced when selecting the value for $\sigma_{\alpha}^{2}$. Zhang and Oliver [21] evaluated the effect of $\sigma_{\alpha}^{2}$ on the screening and determined that $\sigma_{\alpha}^{2}=0.36$ gave results similar to the optimal localization of Furrer and Bengtsson [10]. 
The screening factor $\alpha$ provides an assessment on the reliability of $\hat{\theta}$. Because $\alpha$ is closely related to the inverse of the coefficient of variation, it is clear that a small value of $\alpha$ suggests unreliable correlations which should be reduced in magnitude. Evaluation of $\alpha$, however, cannot be made from a single sample of the variable of interest but instead requires estimation of the squared coefficient of variation for that variable. Bootstrap resampling, as described above, provides a method for estimating $\hat{C}_{v_{i, j}}^{2}$ for any estimator of interest. When $\hat{\theta}$ denotes the Kalman gain, the screening factor $\alpha_{k e}$ is calculated based on $N_{B}$ replicates of Kalman gain, and the screening factor is multiplied to the original estimate of Kalman gain in an element-wise manner:

$K_{e}^{\mathrm{SK}}=\alpha_{k e} \circ K_{e}$

where superscript SK stands for screening Kalman gain. Following the screening of the original Kalman gain matrix, the standard updating (or analysis) step is carried out using $K_{e}^{\mathrm{SK}}$. For later comparison, here we term the EnKF using screened Kalman gain with the short name of EnKF-SKe.

When $\hat{\theta}$ denotes covariances, the screening factors $\alpha_{d d}$ and $\alpha_{y d}$ are calculated, respectively, for $C_{d d}^{f}$ and $C_{y d}^{f}$. Similarly, the screening factors are multiplied to the standard estimates of covariances in an elementwise manner:

$K_{e}^{\mathrm{SC}}=\alpha_{y d} \circ C_{y d}^{f}\left(\alpha_{d d} \circ C_{d d}^{f}+C_{D}\right)^{-1}$

where the superscript SC denotes screening covariance. The consistency between $\alpha_{d d}$ and $\alpha_{y d}$ is an issue to be discussed in later examples. This method is denoted as EnKF-SC.

\section{$51 D$ linear problem}

In this section, we investigate several approaches to the reduction of spurious correlations in data assimilation on a 1 D correlated random field $X=\left\{x_{1}, x_{2}, \ldots, x_{100}\right\}$ with prior mean 0 and exponential covariance as shown in Eq. 14. The covariance function has an exponent of 1.5 , and the range $r$ varies in two testing scenarios,

$C(h)=\exp \left[-3 \cdot(|h| / r)^{1.5}\right]$

where $h=\left|x_{i}-x_{j}\right|$ is the distance between two points.

In this example, the observations are directly the measurements of state variables; thus, the sensitivity $G$ is a matrix that contains 1 at a data location and 0 everywhere else. The true Kalman gain for this problem is computed using the known sensitivity matrix and prior covariance (Eq. 14). To compare the different ensemble-based estimates of Kalman gain with and without screening or localization, an initial ensemble of independent, unconditional realizations are drawn from the same distribution as the prior for $X$.

The distance-dependent localization coefficients matrix $\beta_{y y}$ used in the algorithms of covariance localization and Kalman gain localization is defined using the following equation [10]

$\beta(h)=\frac{1}{1+\left(1+\frac{1}{C(h)^{2}}\right) / N_{e}}$.

A consistent pair of $\beta_{y d}$ and $\beta_{d d}$ is always easy to build for the linear local measurement problem. Because of the simplicity of the sensitivity, $\beta_{y d}$ and $\beta_{d d}$ are simply the block matrices extracted from $\beta_{y y}$ by $\beta_{y d}=\beta_{y y} G^{\mathrm{T}}$ and $\beta_{d d}=G \beta_{y d}$. For bootstrap-based screening covariance methods, the consistency issue is addressed in the single observation test.

In all the following testing scenarios, the number of bootstrapped ensembles, $N_{B}=100$, and all the screening factors $\left(\alpha_{d d}, \alpha_{y d}\right.$, and $\left.\alpha_{k e}\right)$ are calculated based on the same set of bootstrap ensembles. The estimates of the Kalman gain using different localizing or screening algorithms: $K_{e}^{\mathrm{LC}}, K_{e}^{\mathrm{LK}}, K_{e}^{\mathrm{SK}}$, and $K_{e}^{\mathrm{SC}}$ are compared with the true Kalman gain in the following two tests.

\subsection{Single observation}

The range, $r=40$, and the ensemble size, $N_{e}=30$, are used in this example. A single measurement with additive gaussian noise (mean 0 and standard error of 0.05 ) is made at the first gridblock. Hence, the measurement error covariance $C_{D}$ is a scalar and has a value of 0.0025 .

For this linear local measurement problem, the screening covariance satisfies the consistency condition $\left(\alpha_{d d}=G \alpha_{y d}\right)$ as long as the same value of $\sigma_{\alpha}^{2}$ is used for calculating both $\alpha_{y d}$ and $\alpha_{d d}$. Figure 1 shows estimates of Kalman gain from different combinations of $\sigma_{\alpha_{y d}}^{2}$ and $\sigma_{\alpha_{d d}}^{2}$. For the cases of $\sigma_{\alpha_{y d}}^{2} \neq \sigma_{\alpha_{d d}}^{2}$, the estimate of Kalman gain will not be correct at the data location because the consistency condition is violated. Figure $1 b$ shows that when the same value of $\sigma_{\alpha}^{2}$ is used for both covariance matrices, the estimates are good at the data location.

Figure 2 compares the standard estimate of the Kalman gain to the estimates of Kalman gain with screening or distance-based localization applied. Screening and localization both reduce the magnitude of spurious correlations present in the standard estimate 


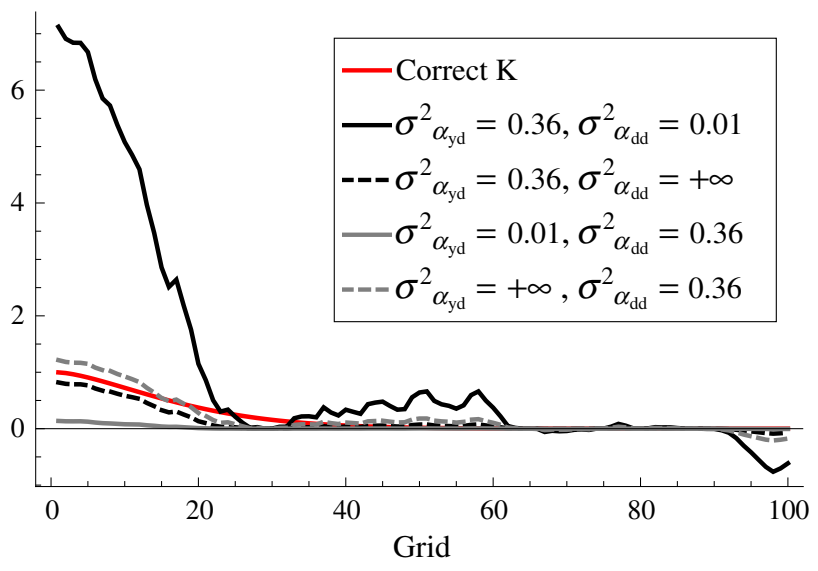

(a)

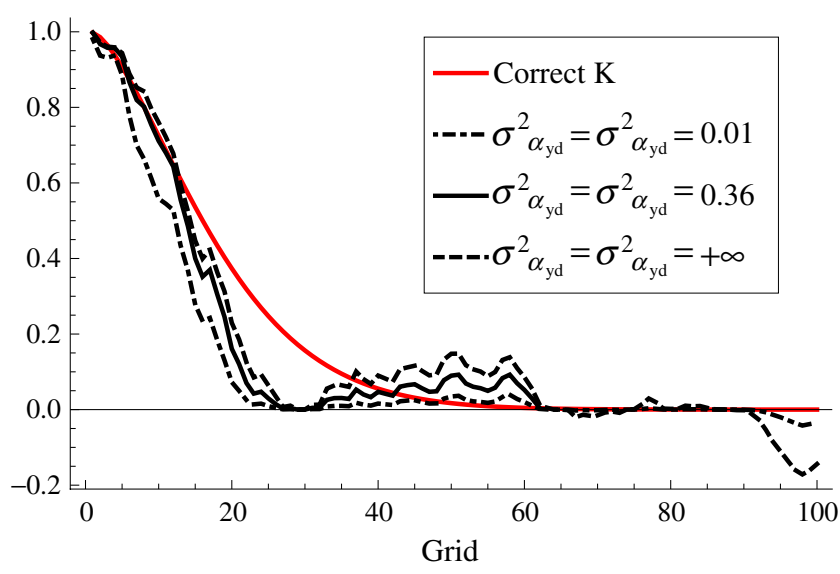

(b)

Fig. 1 Influence and relations of $\sigma_{\alpha_{y d}}^{2}$ and $\sigma_{\alpha_{d d}}^{2}$. a Inconsistent pair. b Consistent pair

of Kalman gain. In this test, since $\beta_{d d}=1.0$, the estimate of Kalman gain from covariance localization $\left(K_{e}^{\mathrm{LC}}\right)$ is exactly the same as the estimate from Kalman gain localization $\left(K_{e}^{\mathrm{LK}}\right)$. The distance-based localization methods completely remove the unreal nonzero values beyond grid 55 due to the zero values of coefficients $\beta_{y d}$ for grids that are located outside the correlation length. Although the bootstrap-based screening algorithms did not completely eliminate the spurious correlations, the magnitudes of the spurious correlations were reduced. Screening Kalman gain performs slightly better than screening covariance in this example.

In order to reduce the influence of randomness on results, this $1 \mathrm{D}$ single observation testing case was evaluated 100 times, each time with different randomly generated initial ensemble. The root mean squared

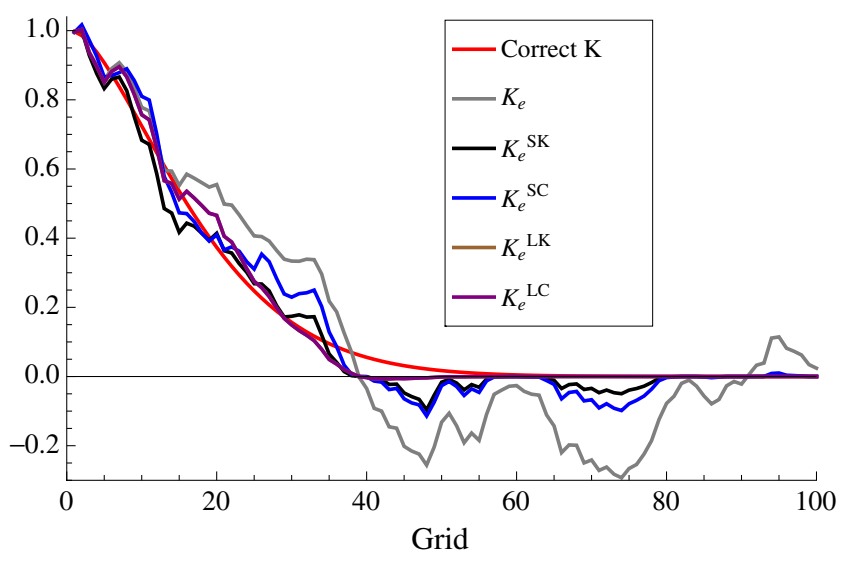

Fig. 2 Estimates of the Kalman gain obtained using five different estimators, compared to the true Kalman gain for the linear 1D problem with a single observation errors (RMSE) for different estimates of Kalman gain are calculated using

$\operatorname{RMSE}_{i}=\sqrt{\frac{\sum_{n=1}^{100}\left(K_{e i, n}-K_{i}\right)^{2}}{100}}$,

where $n$ is the index of the trial and $i$ is the index of the element in the Kalman gain. $K_{e}$ denotes the Kalman gain estimate from different methods, and $K_{i}$ is the true Kalman gain. Figure 3 shows that the distance-based localization methods have the lowest RMSE. Standard EnKF without localization or screening resulted in the highest RMSE values in the region that is distant from data location. Screening the Kalman gain results in smaller RMSE than screening the covariance. Both screening methods, however, have high RMSE values around $x_{20}$. The true Kalman gain or $C_{y d}$ is fairly large at $x_{20}$, and the variability is also large. The screening methods sometimes reduce the true magnitude in that region more than necessary, so the results from the screening methods are slightly worse on average than the standard estimate in that region.

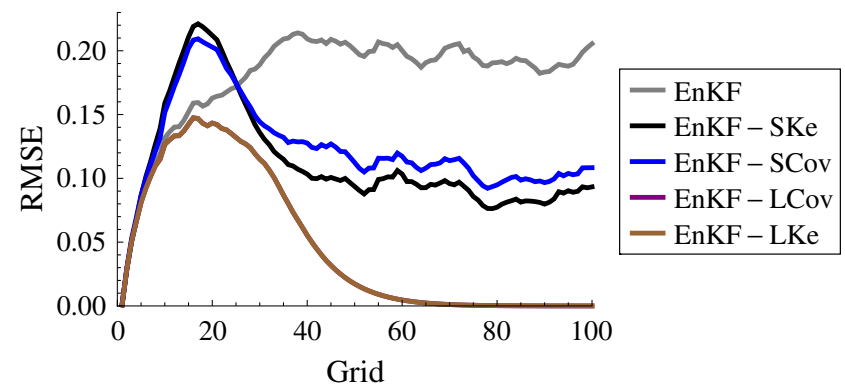

Fig. 3 Root mean squared error (RMSE) of Kalman gain estimates. The curve for EnKF-LKe is identical to the curve for EnKF-LCov in this example 
Figure 4 shows the mean estimate of Kalman gain along with one standard deviation from screening Kalman gain $\left(K_{e}^{\mathrm{SK}}\right)$, screening covariance $\left(K_{e}^{\mathrm{SC}}\right)$, localizing covariance $\left(K_{e}^{\mathrm{LC}}\right)$, and localizing Kalman gain $\left(K_{e}^{\mathrm{LK}}\right)$. Localization and screening result in some bias shown in the expected Kalman gain curve compared to the correct Kalman gain. Due to the effect of distance-based localization, the estimate of Kalman gain obtained from localization methods only shows uncertainty within the correlation length of 40 , beyond which the estimated values exactly go to zero. Screening the covariance shows slightly larger standard deviation than screening the Kalman gain.

To understand why screening the covariance results in greater variability than screening the Kalman gain, we look at the propagations of uncertainty (or error) in these two algorithms. For any function, $f(x, y)$, the linearized approximation to the variance (or error) of $f$ that is propagated from the variances in the scalar variables $x$ and $y$ can be computed as

$\sigma_{f}^{2}=\left(\frac{\partial f}{\partial x}\right)^{2} \sigma_{x}^{2}+\left(\frac{\partial f}{\partial y}\right)^{2} \sigma_{y}^{2}+2 \frac{\partial f}{\partial x} \frac{\partial f}{\partial y} \sigma_{x} \sigma_{y} \rho_{x y}$

where $\rho_{x y}$ is the correlation coefficient between $x$ and $y$ and $\sigma_{x}$ and $\sigma_{y}$ are standard deviations (or standard errors) associated with variables $x$ and $y$, respectively. Using this equation, we can estimate the variances within $K_{e}^{\mathrm{SK}}$ and $K_{e}^{\mathrm{SC}}$ due to the variability in the screening coefficients.

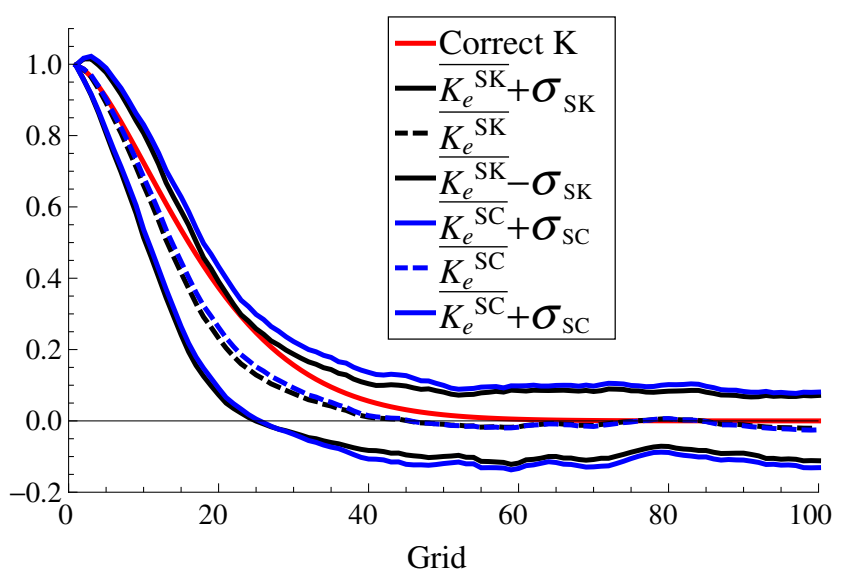

(a)

Fig. 4 Mean estimate of Kalman gain with one standard deviation. a Screening Kalman gain (black), screening covariance (blue). b Localizing Kalman gain (brown), localizing covariance
For any element $i$ in the example with a single observation,

$K_{e, i}^{\mathrm{SK}}=\frac{C_{y d, i}}{C_{d d}+C_{D}} \alpha_{k e, i}$

$K_{e, i}^{\mathrm{SC}}=\frac{C_{y d, i} \alpha_{y d, i}}{C_{d d} \alpha_{d d}+C_{D}}$.

The terms $C_{D}, C_{y d, i}$, and $C_{d d}$ are common in the above two equations, which means they cannot cause the difference of standard deviations shown in Fig. 4a. Thus, we ignore their contributions. $K_{e, i}^{\mathrm{SK}}$ is a function of $\alpha_{k e, i}$, and $K_{e, i}^{\mathrm{SC}}$ is a function of both $\alpha_{y d, i}$ and $\alpha_{d d}$. Following Eq. 17, the relative variance $\tilde{\sigma}_{f}^{2}=\sigma_{f}^{2} / f^{2}$ for $K_{e, i}^{\mathrm{SK}}$ is

$\tilde{\sigma}_{s k, i}^{2}=\frac{\sigma_{\alpha k e, i}^{2}}{\alpha_{k e, i}^{2}}$

Similarly, the relative variance for $K_{e, i}^{\mathrm{SC}}$ is

$\tilde{\sigma}_{s c, i}^{2}=\frac{\sigma_{\alpha_{y d, i}}^{2}}{\alpha_{y d, i}^{2}}+\frac{\sigma_{\alpha_{d d}}^{2}}{\left(\alpha_{d d}+\frac{C_{D}}{C_{d d}}\right)^{2}}-2 \rho\left(\frac{\sigma_{\alpha_{y d, i}}}{\alpha_{y d, i}}\right)\left(\frac{\sigma_{\alpha_{d d}}}{\alpha_{d d}+\frac{C_{D}}{C_{d d}}}\right)$.

As mentioned previously, the variance of $K_{e}$ is not included here, since it is common to both $K_{e}^{\mathrm{SK}}$ and $K_{e}^{\mathrm{SC}}$. Then, the relative variance of $K_{e}^{\mathrm{SK}}$ is only determined by variance of $\alpha_{k e}$ as shown in Eq. 18, while the relative variance of $K_{e}^{\mathrm{SC}}$ is determined by the sum of three error

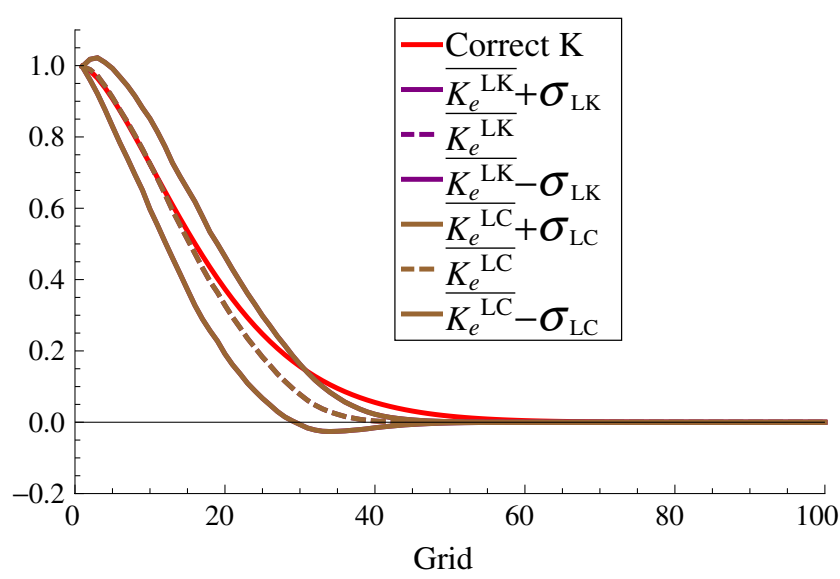

(b)

(purple). The curves for EnKF-LKe and EnKF-LCov are identical in this example 


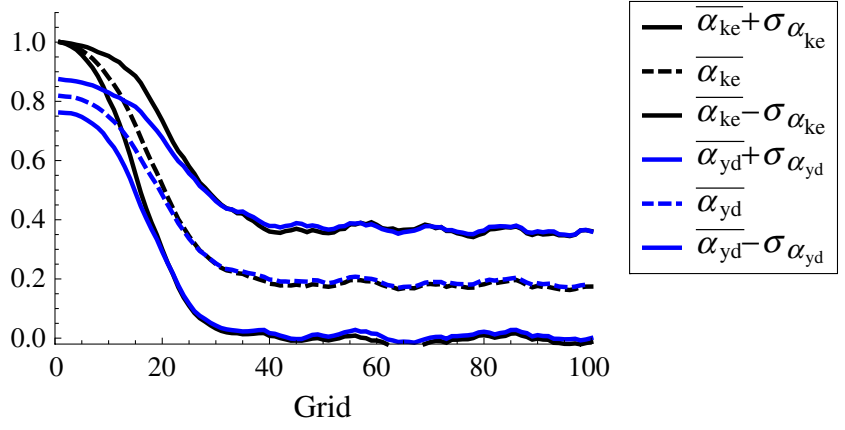

Fig. 5 Mean estimates with one standard deviation for $\alpha_{k e}$ and $\alpha_{y d}$ based on 100 trials

terms (Eq. 19). $\sigma_{\alpha_{d d}}^{2}$ is definitely larger than zero, and $\sigma_{\alpha_{y d}}^{2}$ and $\sigma_{\alpha_{k e}}^{2}$ are comparable in scale as shown in Fig. 5.

In the neighborhood of data location $(i=1), \sigma_{\alpha_{y d}}^{2}$ is significantly larger than $\sigma_{\alpha_{k e}}^{2}$. The reason for $\tilde{\sigma}_{s c, i}^{2} \approx 0$ at data location as shown in Fig. 4a is that $\alpha_{y d, i}=\alpha_{d d}$, $\sigma_{\alpha_{d d}}=\sigma_{\alpha_{y d, i}}$ and the correlation coefficient $\rho=1$ since $\alpha_{y d, i}$ and $\alpha_{d d}$ are perfectly correlated. Moreover, $\frac{C_{D}}{C_{d d}}$ is typically a very small value, which means the absolute value of the negative term approximately equals to the positive terms. The reason for $\tilde{\sigma}_{s k, i}^{2} \approx 0$ at data location is that the Kalman gain value at data location is always approximately 1 as $K_{e, i}=\frac{C_{d d}}{C_{d d}+C_{D}}$ at the data location, which does not change with ensemble. As $i$ increases, however, the standard deviation of $K_{e, i}^{\mathrm{SC}}$ is constantly larger than $K_{e, i}^{\mathrm{SK}}$. This is because the correlation between data and model parameter becomes very small, which leads to the decrease of the correlation coefficient between $\alpha_{y d, i}$ and $\alpha_{d d}$. As $\rho \rightarrow 0, \tilde{\sigma}_{s c, i}^{2} \rightarrow$ $\frac{\sigma_{\alpha_{y d, i}}^{2}}{\alpha_{y d, i}^{2}}+\frac{\sigma_{\alpha_{d d}}^{2}}{\left(\alpha_{d d}+\frac{C_{D}}{C_{d d}}\right)^{2}}$, and the positive term $\frac{\sigma_{\alpha_{d d}}^{2}}{\left(\alpha_{d d}+\frac{C_{D}}{C_{d d}}\right)^{2}}$ is the main contribution for the constant difference between $\tilde{\sigma}_{s c, i}^{2}$ and $\tilde{\sigma}_{s k, i}^{2}$ in the region beyond correlation length.

For multiple data, $\tilde{\sigma}_{s k, i}^{2}$ still only depends on $\sigma_{\alpha_{k e . i}}^{2}$, but $\tilde{\sigma}_{s c, i}^{2}$ is a function of $N_{d}\left(N_{d}+3\right) / 2$ variables $\left(N_{d}\right.$ is the number of data). Estimation of the Kalman gain from screening the covariance is more error sensitive than screening the Kalman gain directly. This conclusion is also true for covariance localization and Kalman gain localization. Although for this 1D single measurement test covariance localization is exactly same as Kalman gain localization, it is not generally the case for multiple data. In addition, while the distance-based localization methods performed better than the screening algorithms for this test, it should be noted that the localization coefficients were calculated based on the true prior covariance, in a problem which was ideal for application of distance-based localization. For more general real problems, these ideal conditions for local- ization do not apply, and distance-based localization can be difficult.

\subsection{Multiple observations}

The screening and localization algorithms all performed well in the test involving a single measurement. In the present scenario, we consider multiple spatially correlated observations for the same 1D model. The main objective is to investigate the impact of influence between different data on the estimates of Kalman gain from EnKF with screening or localization. Five measurements at $x_{1}, x_{25}, x_{50}, x_{75}$, and $x_{100}$ are used for data assimilation. The measurement error covariance $C_{D}$ is a diagonal matrix with the same diagonal values of 0.0025 . The ensemble size is 30 . The range of prior covariance is 100 , so the five measurements are spatially correlated with each other. The true data covariance $C_{d d}^{f}$ is

$$
\left(\begin{array}{cccccc}
1 . & 0.702769 & 0.357364 & 0.148122 & 0.0520728 \\
0.702769 & 1 . & 0.687289 & 0.346227 & 0.142479 \\
0.357364 & 0.687289 & 1 . & 0.687289 & 0.346227 \\
0.148122 & 0.346227 & 0.687289 & 1 . & 0.687289 \\
0.0520728 & 0.142479 & 0.346227 & 0.687289 & 1 .
\end{array}\right)
$$

The true Kalman gain matrix consists of five columns that correspond to the five measurements, respectively. The results from this test can be understood by analyzing the result of any one column from the Kalman gain matrix. Therefore, only the results of column 1 will be presented here.

Figure 6 shows the first column of ensemble-based estimates of Kalman gain from different methods. Screening Kalman gain $\left(K_{e}^{\mathrm{SK}}\right)$ and localizing Kalman gain $\left(K_{e}^{\mathrm{LK}}\right)$ only reduce the magnitude of spurious

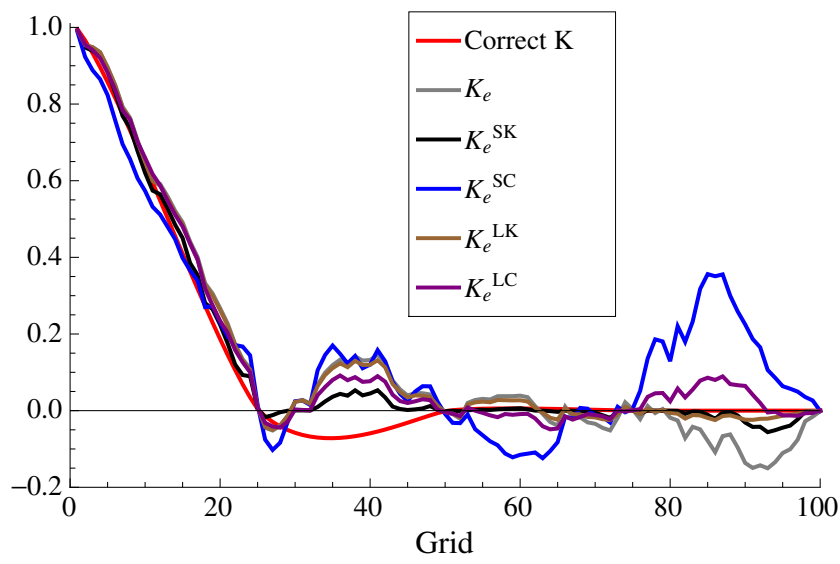

Fig. 6 Estimates of the Kalman gain obtained using five different estimators, compared to the true Kalman gain for the linear 1D problem with multiple observations 


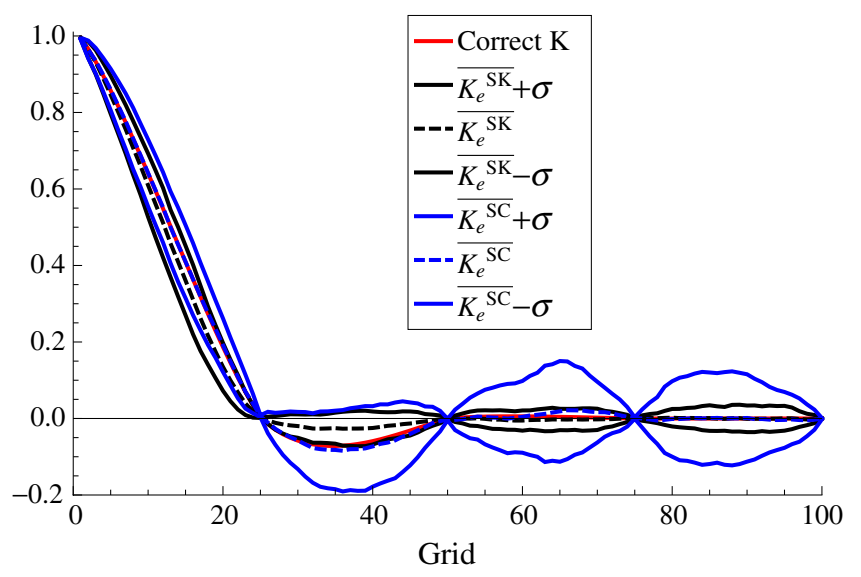

(a)

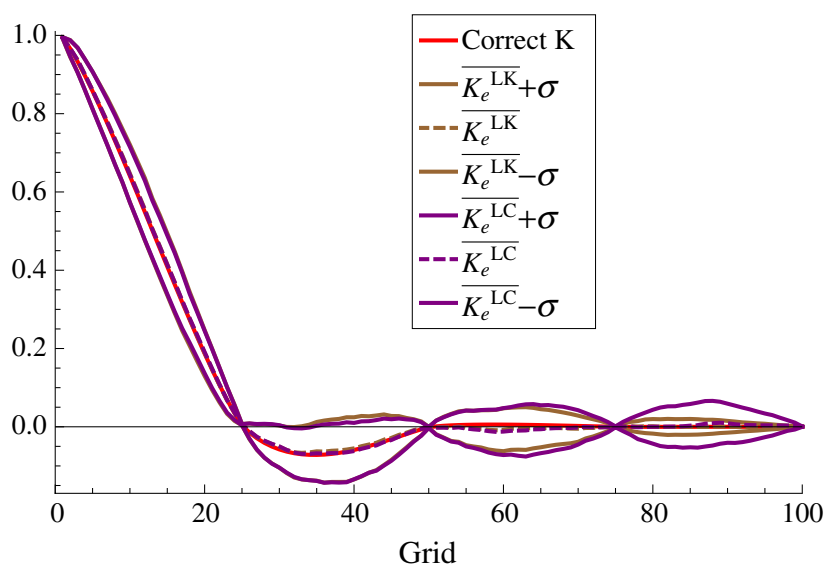

(b)

Fig. 7 Mean estimate of Kalman gain with one standard deviation. a Screening Kalman gain (black), screening covariance (blue). b Localizing Kalman gain (brown), localizing covariance (purple)

correlations and do not change the sign of correlations, screening covariance, and localizing covariance, however, can change the structure of Kalman gain, for example, the negative values are changed to be positive values between grid 80 to grid 100 . It is highly possible that screening/localizing covariance can introduce spurious correlations of larger magnitude. The advantage of screening Kalman gain over localizing Kalman gain is also indicated in Fig. 6. Around grid 40, there are evidently spurious correlations present in the standard estimate of Kalman gain $\left(K_{e}\right)$ of opposite sign to the true correlations shown in correct Kalman gain. The screening Kalman gain $\left(K_{e}^{\mathrm{SK}}\right)$ decreased the magnitudes, but the localizing Kalman gain $\left(K_{e}^{\mathrm{LK}}\right)$ had no effect. As the correlation length is 100 for this test, the values of distance-based localization coefficients around grid 40 are high, between 0.85 and 0.95 ; therefore, localization cannot eliminate the spurious correlations shown in the high correlation region.

In order to obtain reliable statistical conclusions, the test was repeated 100 times with different initial ensembles. Figure 7 shows the mean estimates of Kalman gain with one standard deviation. The estimate from screening the Kalman gain has significantly smaller standard deviation than the estimate from screening the covariance. Similarly, the result from localizing the Kalman gain has smaller standard deviation than from localizing the covariance, especially between grid 80 and grid 100. Figure 8 shows the RMSE of estimates of Kalman gain from the different methods. Only in the region (between grid 1 and grid 20) where data and model parameters are most highly correlated, screening/localizing covariance results in smaller RMSE values than screening/localizing Kalman gain. In the rest of the region, screening/localizing covariance results in higher RMSE than screening/localizing Kalman gain, which is consistent with the conclusion from the error analysis that the canceling effect of negative crossing terms vanishes as the correlation coefficients get close to zero. The RMSE values are nearly zero at data locations because the measurements are directly of the model variables. The rows of $C_{d d}$ are identical to the rows of $C_{y d}$ corresponding to the five data locations, thus the values of the Kalman gain at the five data locations obtained from $C_{d d}\left(C_{d d}+C_{D}\right)^{-1}$, which is approximately an identity matrix when the magnitudes of the entries in $C_{D}$ are very small. Thus, regardless of the values in covariances $C_{d d}$ or $C_{y d}$, the values of the 1 st column of Kalman gain matrix at five data locations are approximately $1,0,0,0$, and 0 , respectively. If we neglect the sharp decrease in RMSE values at data locations, the RMSE values from the screening Kalman gain become smaller as the distance from $x_{1}$ increases.

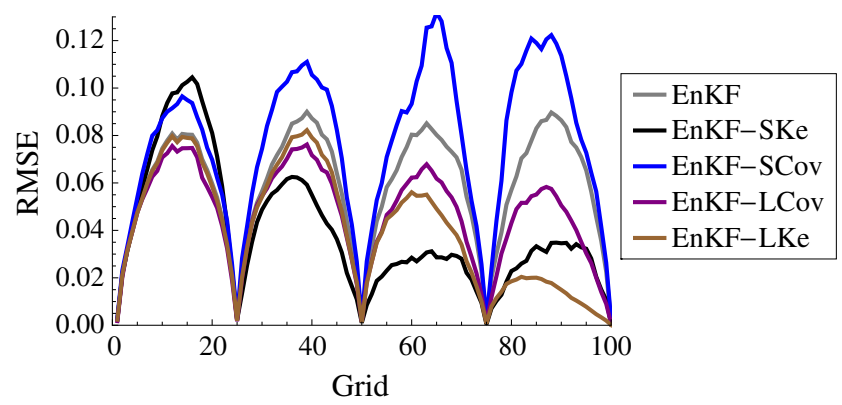

Fig. 8 Root mean squared error of Kalman gain estimates 
On the other hand, screening covariance shows an opposite trend.

In order to see to what extent the screening/localizing algorithms are influenced by the ensemble size $\left(N_{e}\right)$ or the number of bootstrap samples $\left(N_{B}\right)$, a simple sensitivity study was performed. The criterion for quantifying the influence is the mean RMSE computed using Eq. 20. Figure 9a shows the logarithm of $\overline{\mathrm{RMSE}}$ versus ensemble size $N_{e}$. The tested ensemble size is gradually increased from 5 to 200 by a step size of 5 . When $N_{e}=5$, screening Kalman gain has the smallest $\overline{\mathrm{RMSE}}$ value. When $N_{e} \geq 100, \overline{\mathrm{RMSE}}$ is small for all methods. The $\overline{\mathrm{RMSE}}$ of the estimate of the Kalman gain is smaller from screening the Kalman gain than that obtained from screening the covariance for all different ensemble sizes. Localizing the Kalman gain also results in slightly smaller $\overline{\mathrm{RMSE}}$ than localizing the covariance. Figure $9 \mathrm{~b}$ shows how the screening methods are influenced by the number of bootstrap samples for $N_{B}$ from 5 to 200. Screening Kalman gain is not very sensitive to $N_{B}$, while screening covariance seems to be influenced by $N_{B}$, only when $N_{B}$ is very small. The $\overline{\mathrm{RMSE}}$ values from screening covariance are consistently higher than those from screening Kalman gain.

$\overline{\mathrm{RMSE}}=\sqrt{\frac{\sum_{i=1}^{N_{y}}\left(K_{e, i}-K_{i}\right)^{2}}{N_{y}}}$

In the 1D linear problem with single/multiple observations, we were able to use the true prior covariance and true range in the localization function. In sequential data assimilation, this may not be the case, since the prior covariance changes with time due to the assimilated data at each timestep [4]. Thus, in the next section, a comparison study is carried on a sequential data assimilation on a nonlinear $2 \mathrm{D}$ reservoir flow model.

\section{$62 D$ highly nonlinear problem}

\subsection{Reference model}

Reference data for evaluation of the methods are generated from a reference reservoir model that is $100 \times$ 100 with individual gridblock dimensions of $30 \times 30 \times$ $20 \mathrm{ft}$. The wells are drilled in a repeat five-spot water flooding pattern. There are 15 producers and 15 injectors in the field. Porosity is 0.20 for all gridblocks. The only uncertain model parameter in this problem is $\log$ permeability at each grid block. The reference $\log$ permeability field is generated using an isotropic exponential variogram model with a practical range of 10 gridblocks, mean of 3.5 , and standard deviation of 1.0. Figure 10 shows the reference log permeability field with black circles denoting the locations of production wells and triangles denoting the locations of water injection wells.

The producers are controlled by fixed bottom hole pressure with maximum oil production rate as the secondary constraint. The injectors are controlled by fixed water injection rate with maximum bottom hole pressure as the secondary constraint. The reference reservoir model is produced for a total of 520 days. Figure 11 shows the production data profiles from the reference model which include the oil and water production rates for the producers as well as the bottom hole pressure of the injectors.

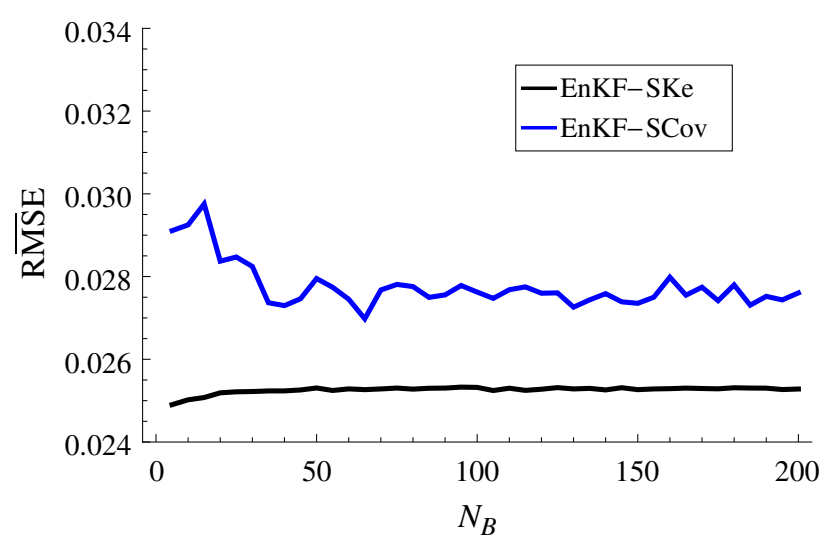

(b)

Fig. 9 Investigation of the sensitivity of RMSE to ensemble size, $N_{e}$, and to number of bootstrap replicates, $N_{B}$ (based on 40 evaluations). a $N_{e}$ influence ( $N_{B}=100$ for screening methods). b $N_{B}$ influence $\left(N_{e}=200\right)$ 


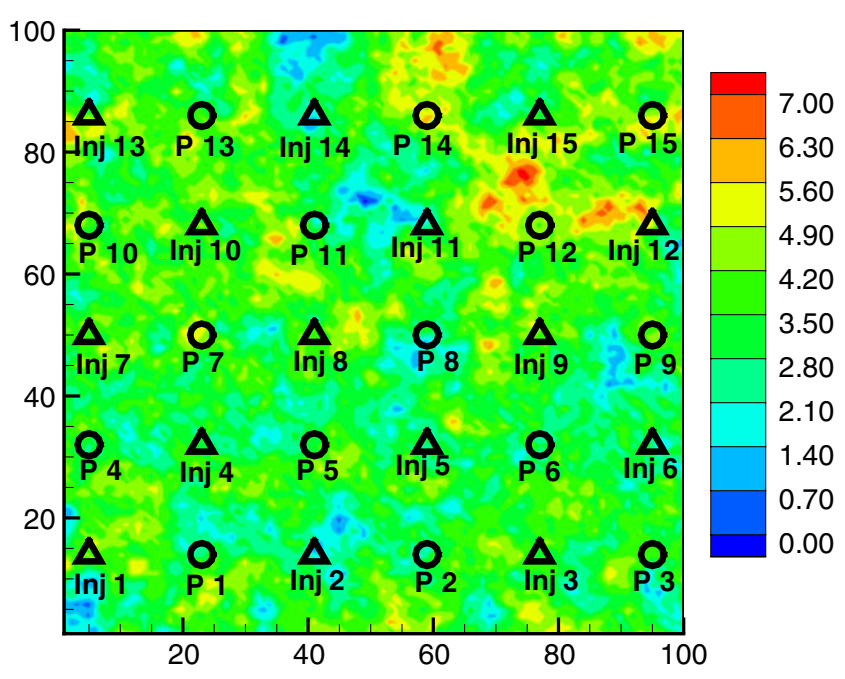

Fig. 10 The reference log permeability field

\subsection{Test setup}

The total production period for the reservoir model is 520 days. The time between day 0 and day 250 is treated as the production history and the period from day 251 to day 520 is considered as the prediction period. Water injection in the field started from day 0 and continued until the end of the production period (520 days). The oil and water production rate data from each producer and the bottom hole pressure data from each injector are used as observations during data assimilation. The observations are taken at day 10 and every 60 days thereafter until day 250. Thus, there are a total of five data assimilation time steps and 45 production data at each assimilation step. The measurement noise for the injector bottom hole pressure and oil production rate are assumed to have a mean of 0 , and the standard deviation of measurement error is assumed to be $1 \%$ of the actual observation value. The standard deviation of measurement error for water production rate data is assumed to be $2 \%$ of the actual observation value.
In order to verify the ability of different screening and localizing algorithms for eliminating spurious correlations, a small ensemble containing 30 members was used, which is likely to result in significant sampling errors. There are a total of 225 data to be assimilated during five data assimilation steps using this small ensemble. Log permeability, pressure, and water saturation are included into the state vector. Thus, the state vector for each ensemble member contains a total of 30,000 model parameters and state variables.

For this high-dimensional data assimilation problem, the measurements are nonlocal. A consistent covariance localization involves computing the full covariance matrix. To avoid the intensive cost of computing the full covariance matrix, Chen and Oliver [4] proposed an approximate form for constructing $\beta_{d d}$ by replacing the full covariance matrix, $\beta_{y y}$, with an identity matrix.

$\beta_{d d}=\beta_{y d}^{\mathrm{T}} \beta_{y d}$

The authors also showed that acceptable results were obtained by using the proposed approximation. Therefore, in this test, Eq. 21 is used for computing $\beta_{d d}$, and $\beta_{y d}$ is built using Eq. 15 with a range of 25 gridblocks that is determined based on the correlation length of the prior log permeability field together with the sensitivity and well pattern information. The same localization function is used for all three types of data. Both log permeability and dynamic state variables (including water saturation and pressure) are updated with localization.

For the two bootstrap-based screening algorithms, $N_{B}=50, \sigma_{\alpha}^{2}=0.36$, and the same random seed is used during bootstrapping. Two more cases were also evaluated for comparison, including the standard EnKF with a small ensemble size of 30 and that with a fairly large ensemble size of 2,000. For this complex flow model, we do not know the exact Kalman gain so the estimate of Kalman gain from EnKF with $N_{e}=2,000$ is
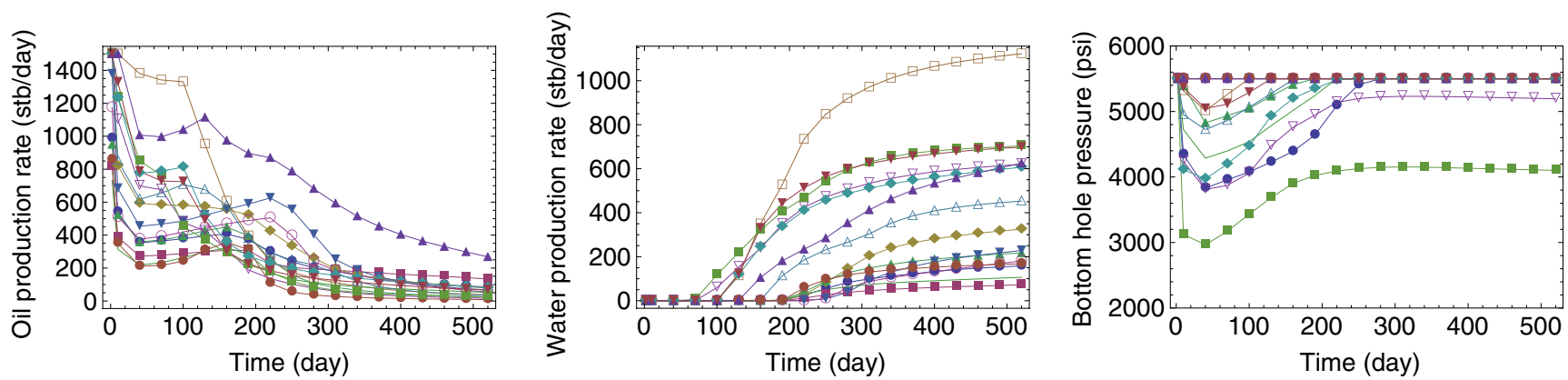

Fig. 11 The production profiles from the reference model (different curves denote different wells) 
used for comparison with the estimates from the other methods.

\subsection{Results and analysis}

\subsubsection{Match production data}

The variability represented by the initial ensemble is able to cover most of the observations from the reference model. As an example, Fig. 12a shows the predictions of oil production rate of producer $\mathrm{P} 9$ from the initial ensemble prior to assimilating any observations. After assimilating 45 data at the first data assimilation step, the updated ensemble from the standard EnKF loses nearly all the ensemble variability as shown in Fig. 12b, c, which illustrate the necessity of applying screening or localization algorithms.

Once the entire data assimilation process is complete, the final updated ensemble of log permeability was evaluated from the beginning (day 0) up to the end of the production period (day 520) using a commercial reservoir simulator [19]. Figure 13 shows the predictions of different production data for three wells obtained by rerunning the final updated log permeability fields from day 0 to day 520. The standard EnKF without covariance/Kalman gain regularization is not able to match the production profiles because of the ensemble collapse that was observed at early data assimilation timesteps. The EnKF with screening covariance (EnKF-SC) shows larger ensemble variability but does not match data well. The remaining three methods (EnKF-SKe, EnKF-LKe, and EnKF-LCov) have comparatively good matches to the reference production data from the reference model. In order to make a quantitative evaluation of the data matches from different methods, two evaluation criteria are defined including the average root mean squared error $\hat{e}_{d}$

$\hat{e}_{d}=\frac{1}{N_{t} N_{w}} \sum_{t=1}^{N_{t}} \sum_{w=1}^{N_{w}} \sqrt{\frac{1}{N_{e}} \sum_{i=1}^{N_{e}} \frac{\left(d_{t, w}^{\mathrm{obs}}-d_{t, w, i}\right)^{2}}{\sigma_{t, w}^{2}}}$

and the average prediction spread $\hat{\sigma}_{d}$

$\hat{\sigma}_{d}=\frac{1}{N_{t} N_{w}} \sum_{t=1}^{N_{t}} \sum_{w=1}^{N_{w}} \sqrt{\frac{1}{N_{e}} \sum_{i=1}^{N_{e}}\left(d_{t, w, i}-\left\langle d_{t, w}\right\rangle\right)^{2}}$

where $N_{e}$ is the number of ensemble members, $N_{t}$ is the number of data records, $N_{w}$ is the number of wells for the same type of data, $d_{t, w}^{\text {obs }}$ denotes observation, $d_{t, w, i}$ denotes predicted data and $\sigma_{t, w}^{2}$ denotes the observation error, and $\left\langle d_{t, w}\right\rangle$ denotes the mean of ensemble prediction. Table 1 shows the $\hat{e}_{d}$ versus $\hat{\sigma}_{d}$ for three types of data. The standard EnKF results in the largest $\hat{e}_{d}$ and smallest $\hat{\sigma}_{d}$, which is the worst case followed by EnKF with screening covariance (EnKF-SC). The other three methods result in similar $\hat{e}_{d}$ for all data types, while EnKF-SKe has relatively smaller $\hat{\sigma}_{d}$ than EnKF-LKe and EnKF-LCov.

\subsubsection{Estimates of model parameter (log permeability)}

Figure 14 shows the final estimates (ensemble mean) of $\log$ permeability obtained from different methods. The estimate of log permeability from the standard EnKF $\left(N_{e}=30\right)$ shows extremely high and low values. For EnKF-SCov, the estimates also suffer from the overshooting issue and are highly discontinuous, probably as a result of inconsistency in screening the covariance matrices. The other three methods result in better estimates of log permeability as the magnitudes of

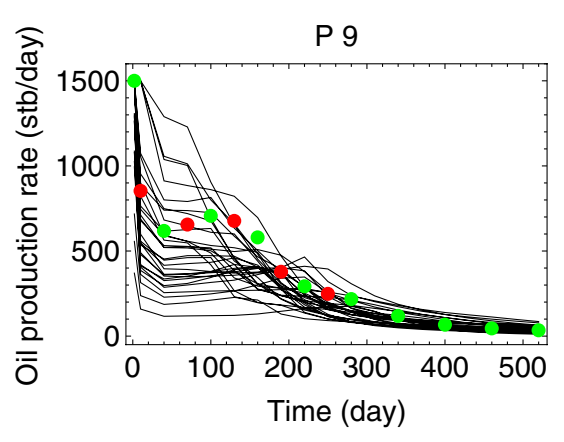

(a)

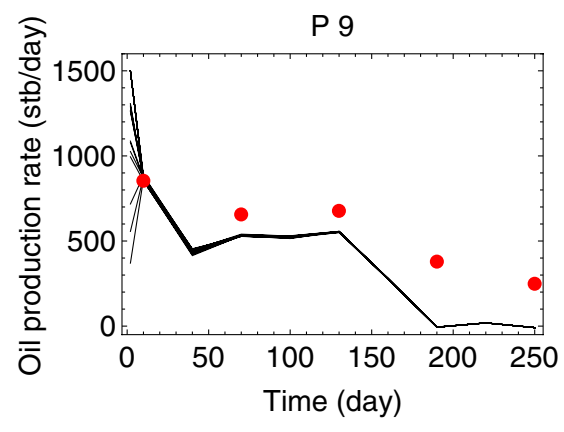

(b)

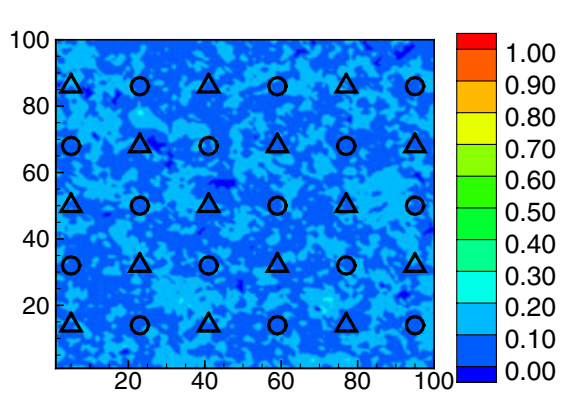

(c)
Fig. 12 The loss of ensemble variability for standard EnKF with an ensemble size of 30 (red dots denote observations used for data assimilation, green dots denote observations only for comparison, black curves denote ensemble outputs in $\mathbf{a}$ and $\mathbf{b}$ ). a Initial. b During data assimilation. c The ensemble STD of log permeability after 1 st data assimilation 

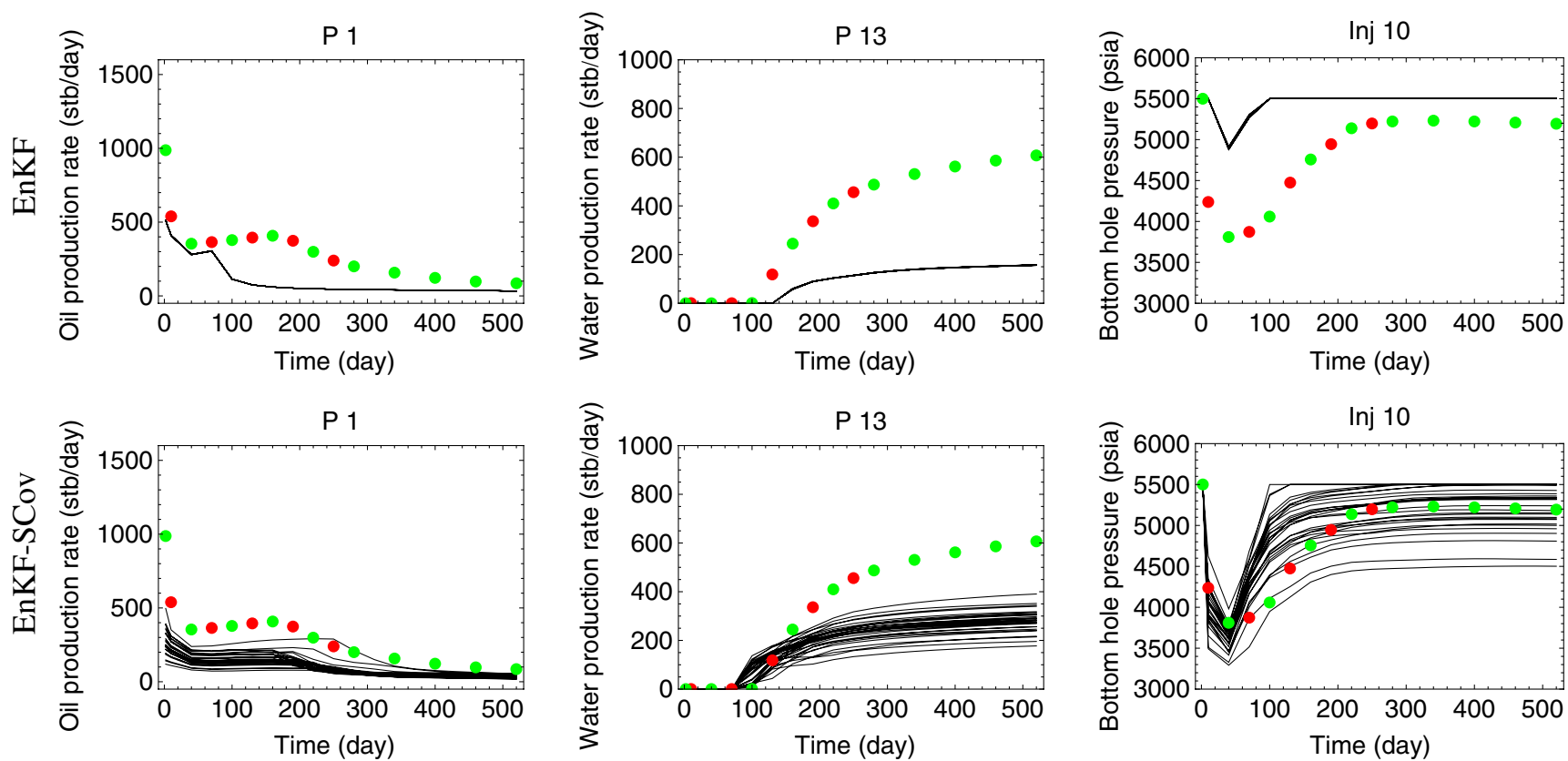

Inj 10
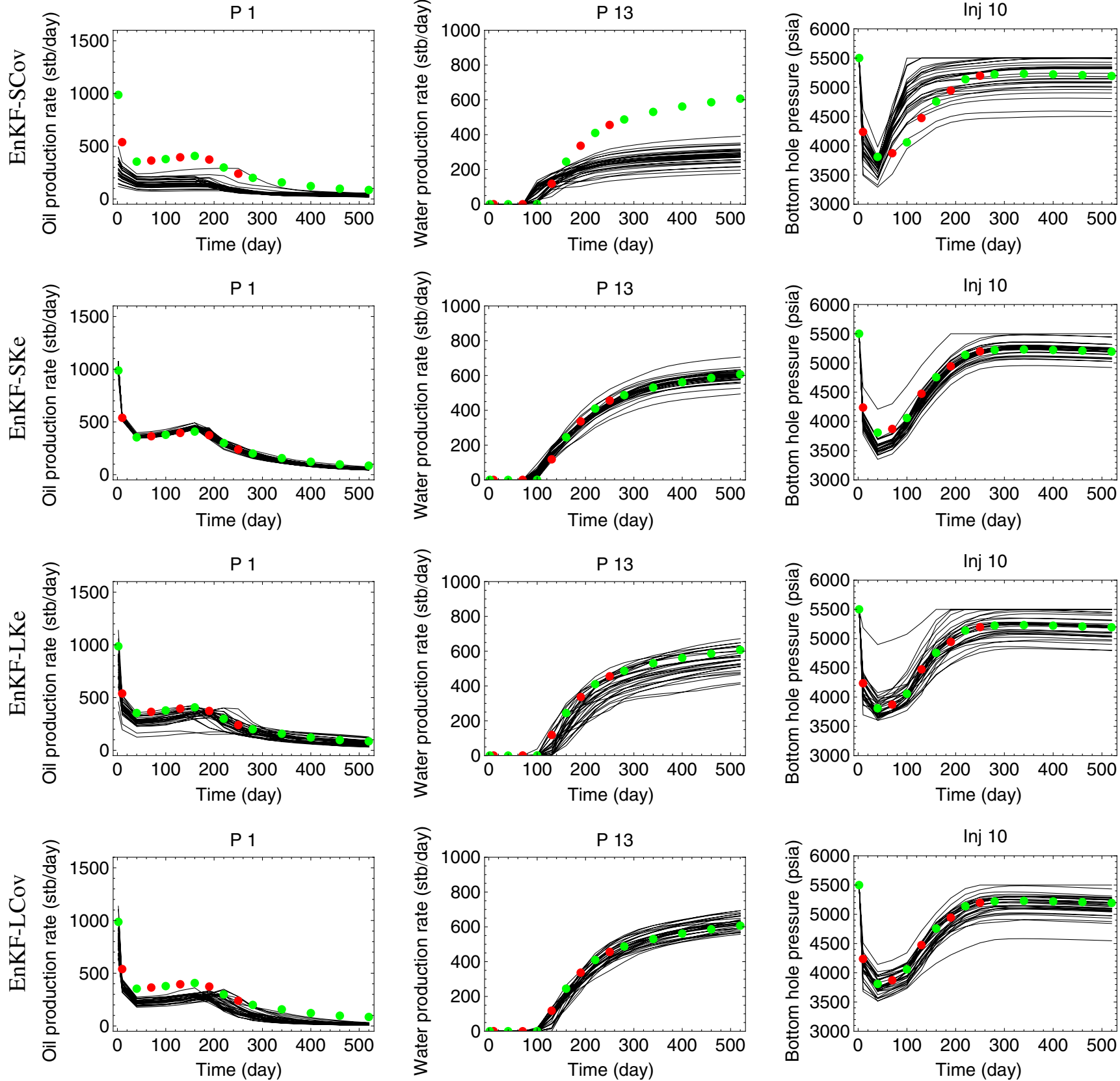

Fig. 13 Ensemble predictions based on final estimated log permeability fields for wells P 1, P 13, Inj 10: observations used for data assimilation (red dots), observations only for comparison (green dots), ensemble outputs (black lines) 
Table 1 The average error versus average spread $\left(\hat{e}_{d} / \hat{\sigma}_{d}\right)$ of the predictions of three types of data

\begin{tabular}{llcc}
\hline & OPR & WPR & BHP \\
\hline EnKF $\left(N_{e}=30\right)$ & $49 / 0.6$ & $2,049 / 0.7$ & $11 / 2.2$ \\
EnKF-SCov & $56 / 33$ & $256 / 24$ & $5 / 32$ \\
EnKF-SKe & $24 / 19$ & $124 / 16$ & $3 / 51$ \\
EnKF-LKe & $26 / 46$ & $130 / 29$ & $2 / 75$ \\
EnKF-LCov & $37 / 39$ & $105 / 27$ & $2 / 67$ \\
\hline
\end{tabular}

$O P R$ oil production rate, $W P R$ water production rate, $B H P$ bottom hole pressure

estimates are similar to those of the reference model. There are, however, some artificial phenomena appearing in the estimate from EnKF-LCov as the log permeability values around the production well $\mathrm{P} 2$ $(x=59, y=14)$ appear significantly different in magnitudes from the region outside the neighborhood of well $\mathrm{P} 2$. The inconsistency issue might be responsible for this behavior, although it is not so severe as shown in the case of EnKF-SCov. Table 2 shows the quantitative evaluation of the estimates of log permeability obtained from different methods. Spatial mean is the average value of log permeability over all gridblocks. The true spatial mean has a value of 3.5 , so two methods including EnKF-SKe and EnKF $\left(N_{e}=2,000\right)$ provide accurate estimates of the spatial mean. The ensemble STD is the average of grid-based ensemble standard de-
Table 2 Statistical quantities of the final estimates of $\log$ permeability

\begin{tabular}{llll}
\hline & Ensemble mean & Ensemble STD & RMSE \\
\hline EnKF $\left(N_{e}=30\right)$ & 3.53 & 0.005 & 2.98 \\
EnKF-SCov & 3.21 & 0.04 & 4.41 \\
EnKF-SKe & 3.50 & 0.15 & 1.25 \\
EnKF-LKe & 3.65 & 0.95 & 1.5 \\
EnKF-LCov & 3.53 & 0.72 & 1.24 \\
EnKF $\left(N_{e}=2,000\right)$ & 3.50 & 0.87 & 1.23 \\
\hline
\end{tabular}

viation of log permeability. Compared to the ensemble STD obtained from EnKF $\left(N_{e}=2,000\right)$, the ensemble STD from EnKF-LKe is high, while the values from EnKF $\left(N_{e}=30\right)$ and EnKF-SCov are low. RMSE is the average grid-based root mean squared error of estimates of log permeability. The RMSE values from EnKF-SKe and EnKF-LCov are similar to the RMSE value obtained from $\operatorname{EnKF}\left(N_{e}=2,000\right)$.

\subsubsection{The estimates of Kalman gain}

The Kalman gain contains the weighted correlations between data and variables in the state vector. For this $2 \mathrm{D}$ problem, there are 135 (45 data $\times 3$ types of model variables) different weighted correlation maps at each data assimilation step. Comparing each of them is
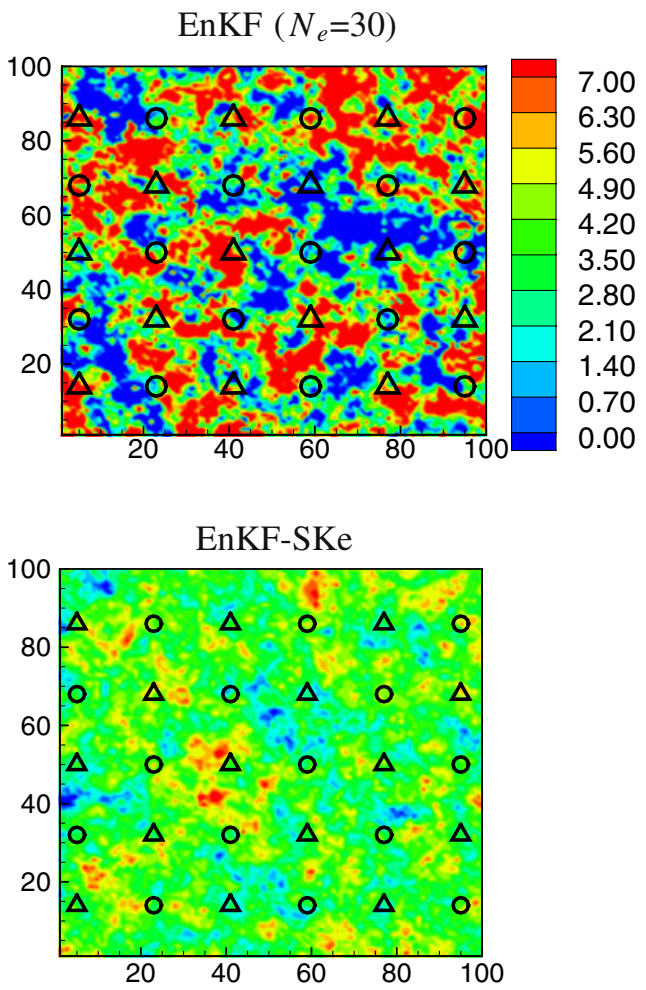
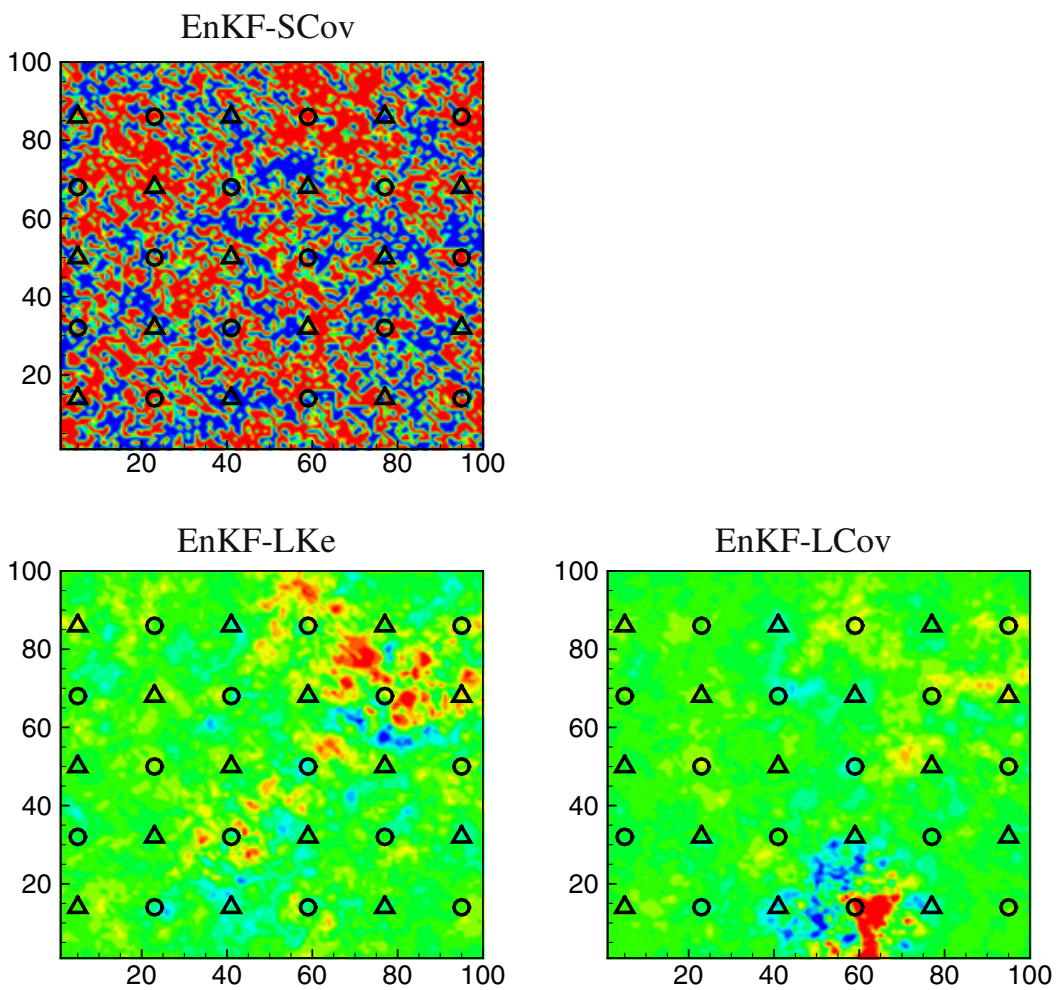

Fig. 14 Final estimates of log permeability 
Table 3 Average RMSE of the Kalman gain estimates at data assimilation time 1

\begin{tabular}{llllll}
\hline Variable/data & EnKF $\left(N_{e}=30\right)$ & EnKF-SCov & EnKF-SKe & EnKF-LKe & EnKF-LCov \\
\hline $\ln K / \mathrm{OPR}$ & 0.0020 & 0.0008 & 0.0008 & 0.0008 & 0.0007 \\
$\ln K / \mathrm{BHP}$ & 0.0004 & 0.0001 & 0.0001 & 0.0001 & 0.00006 \\
$P / \mathrm{OPR}$ & 0.65 & 0.57 & 0.50 & 0.53 & 0.57 \\
$P / \mathrm{BHP}$ & 0.1 & 0.05 & 0.03 & 0.03 & 0.02 \\
\hline
\end{tabular}

time-consuming. The effect of spurious correlations should be small in an ensemble of size 2,000 which provides a good basis for comparison. Thus, the estimate of the Kalman gain from the EnKF with $N_{e}=2,000$ is treated as the true Kalman gain, and the RMSE of estimates from other methods are computed. The Kalman gain matrix is composed of nine block matrices whose dimensions are $10,000 \times 15$ as shown in Eq. 24 .

$K_{e}=\left[\begin{array}{ccc|ccc|ccc}\ln K_{1} / \mathrm{OPR}_{1} & \ldots & \text { /OPR } 15 & \text { /WPR } 1 & \ldots & \text { /WPR } 15 & / \text { BHP } 1 & \ldots & / \text { BHP } 15 \\ \vdots & \vdots & \vdots & \vdots & \vdots & \vdots & \vdots & \vdots & \vdots \\ \ln K_{10,000 / \text { OPR } 1} & \ldots & \ldots & \ldots & \ldots & \ldots & \ldots & \ldots & \ldots \\ \hline P_{1} / \text { OPR } 1 & \ldots & \ldots & \ldots & \ldots & \ldots & \ldots & \ldots & \ldots \\ \vdots & \vdots & \vdots & \vdots & \vdots & \vdots & \vdots & \vdots & \vdots \\ P_{10,000 / \text { OPR } 1} & \ldots & \ldots & \ldots & \ldots & \ldots & \ldots & \ldots & \ldots \\ \hline S_{w_{1} / \text { OPR } 1} & \ldots & \ldots & \ldots & \ldots & \ldots & \ldots & \ldots & \ldots \\ \vdots & \vdots & \vdots & \vdots & \vdots & \vdots & \vdots & \vdots & \vdots \\ S_{w_{10,000} / \text { OPR } 1} & \ldots & \ldots & \ldots & \ldots & \ldots & \ldots & \ldots & \ldots\end{array}\right]$.

where $\ln K$ is $\log$ permeability, $P$ is pressure, $S_{w}$ is water saturation, OPR is the oil production rate of a producer, WPR is the water production rate of a producer, and BHP is the bottom hole pressure of an injector. An average RMSE of each block matrix with respect to the Kalman gain obtained from the standard EnKF with $N_{e}=2,000$ is calculated at two representative times to evaluate the quality of the Kalman gain estimate for a particular type of data and model variable.

For the early time, before water arriving at producers from injectors (mainly the first two data assimilation times), the estimates of $C_{d d}^{f}$ are diagonal dominant because the data are highly correlated with other data.
Table 3 shows the average RMSE of the Kalman gain estimates at data assimilation time 1 . Because the observed values of WPR are nearly zero, the average RMSE values are not calculated for the blocks related to either WPR or $S_{w}$. The standard EnKF has the highest average RMSE values for the estimates of the Kalman gain. The other methods provide comparatively good estimates of the Kalman gain.

By data assimilation time 4 , most of the producers from the reference model show significant water production and the correlations between different data become stronger. Table 4 shows the average RMSE of the Kalman gain estimates at data assimilation time 4. In this table, the results from the standard EnKF
Table 4 Average RMSE of the Kalman gain estimates at data assimilation time 4

\begin{tabular}{lclcc}
\hline & EnKF-SCov & EnKF-SKe & EnKF-LKe & EnKF-LCov \\
\hline $\ln K / \mathrm{OPR}$ & 0.04 & 0.001 & 0.002 & 0.001 \\
$\ln K / \mathrm{WPR}$ & 5.4 & 0.01 & 0.2 & 1.1 \\
$\ln K / \mathrm{BHP}$ & 0.002 & 0.0002 & 0.0002 & 0.0002 \\
$P / \mathrm{OPR}$ & 12.14 & 0.67 & 0.72 & 0.69 \\
$P / \mathrm{WPR}$ & $1,629.1$ & 3.3 & 36.7 & $1,726.9$ \\
$P / \mathrm{BHP}$ & 0.58 & 0.08 & 0.07 & 0.09 \\
$S_{w} / \mathrm{OPR}$ & 0.0038 & 0.0002 & 0.0002 & 0.0002 \\
$S_{w} / \mathrm{WPR}$ & 0.5 & 0.001 & 0.01 & 0.09 \\
$S_{w} / \mathrm{BHP}$ & 0.0002 & 0.00002 & 0.00002 & 0.00002 \\
\hline
\end{tabular}




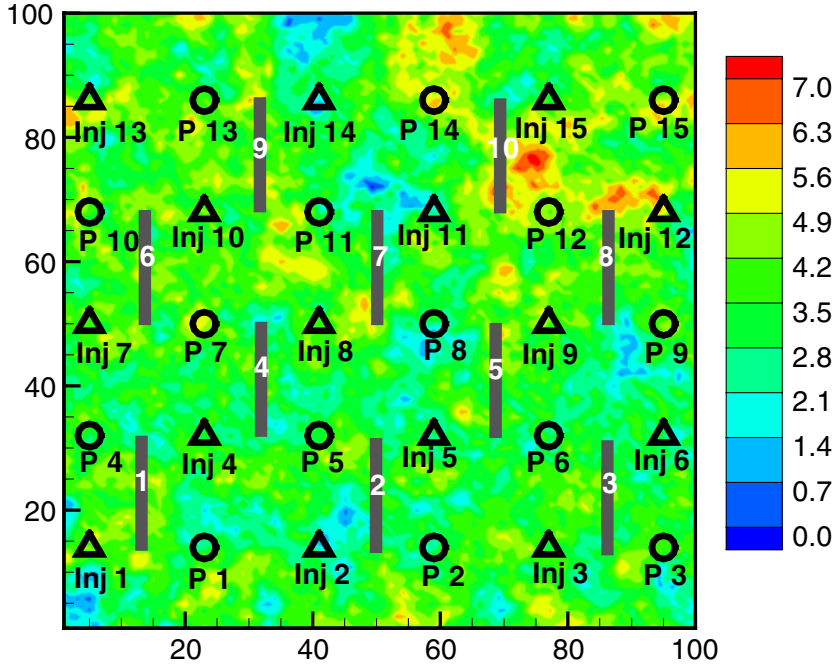

Fig. 15 Reference log permeability field with 10 faults

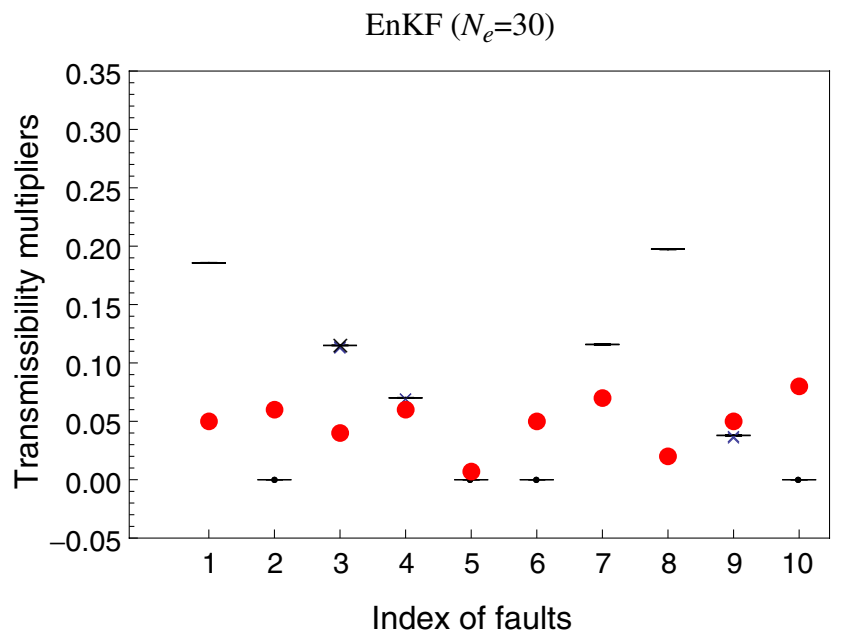

EnKF-LKe

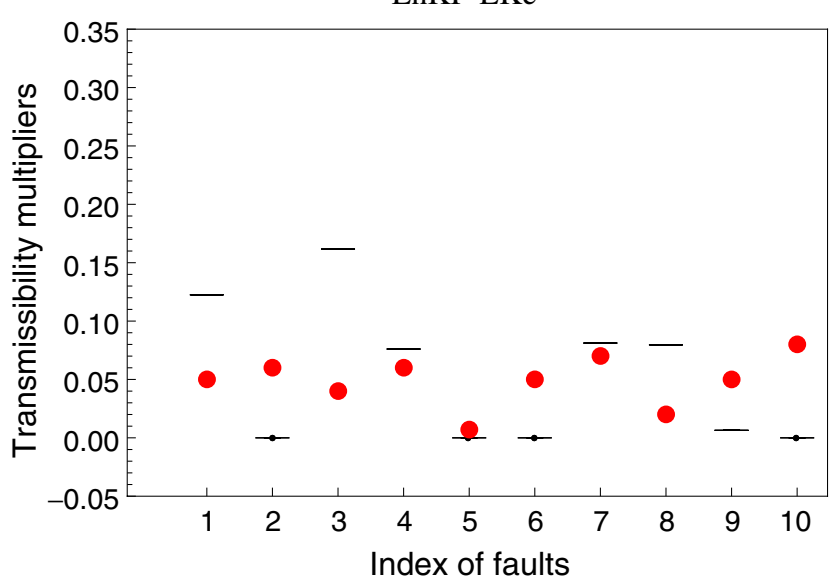

$\left(N_{e}=30\right)$ are not included because the updated ensemble from the EnKF lost almost all the ensemble variability at the 1st data assimilation time and the estimates of the Kalman gain at later assimilation times do not contain any information. Comparing these four methods in Table 4, EnKF-SCov shows much larger RMSE values than those obtained from the other three methods, and EnKF-SKe generally results in the lowest RMSE values, especially for $\ln K / \mathrm{WPR}, P / \mathrm{WPR}$, and $S_{w} / \mathrm{WPR}$.

\subsection{Simultaneous estimation of spatially correlated and uncorrelated model parameters}

In the previous reservoir data assimilation example, we estimated log permeability, which is a spatially correlated model parameter for which distance-dependent localization might be expected to work well. There are, however, sometimes model parameters to be estimated for which the concept of distance is not meaningful. In
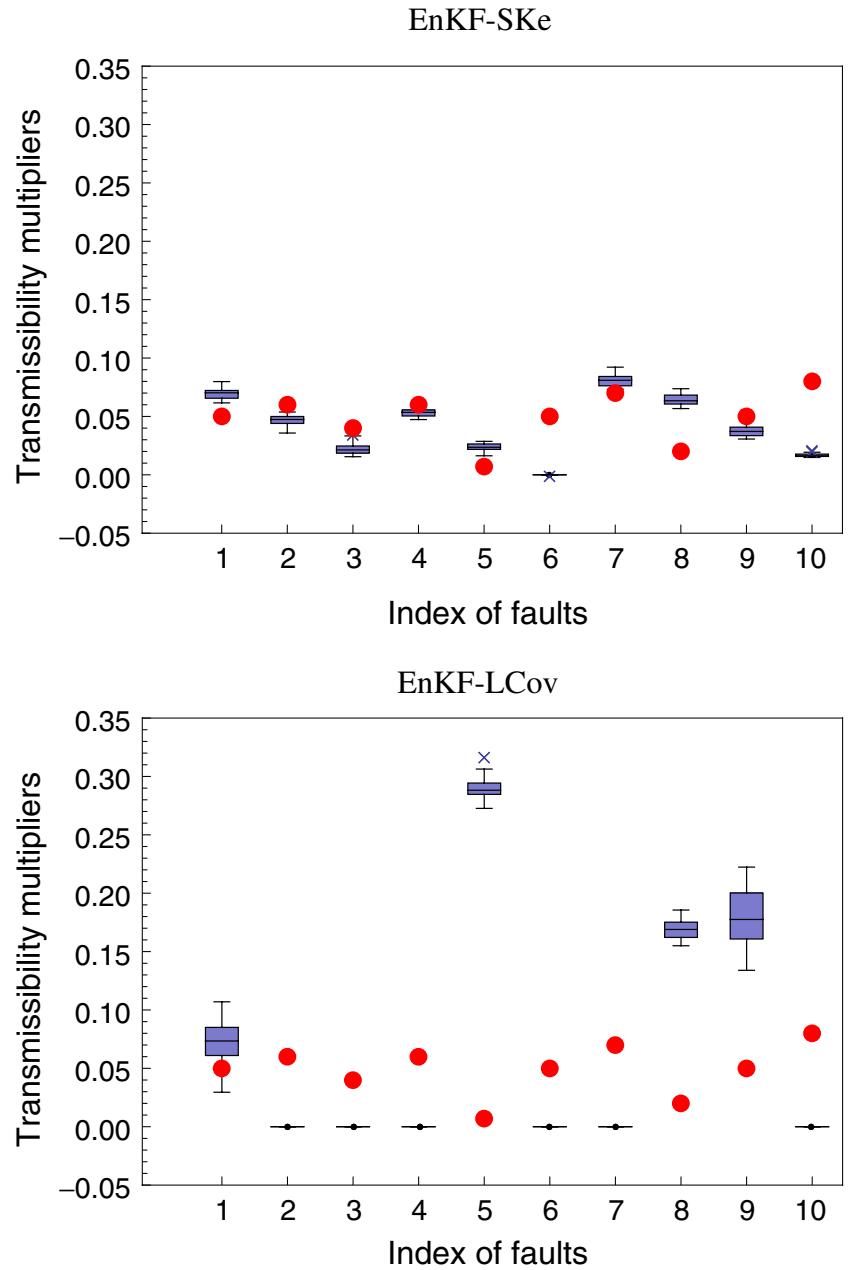

Fig. 16 Final updated transmissibility multipliers (in the whisker box plot, red dots denote the true transmissibility multipliers) 


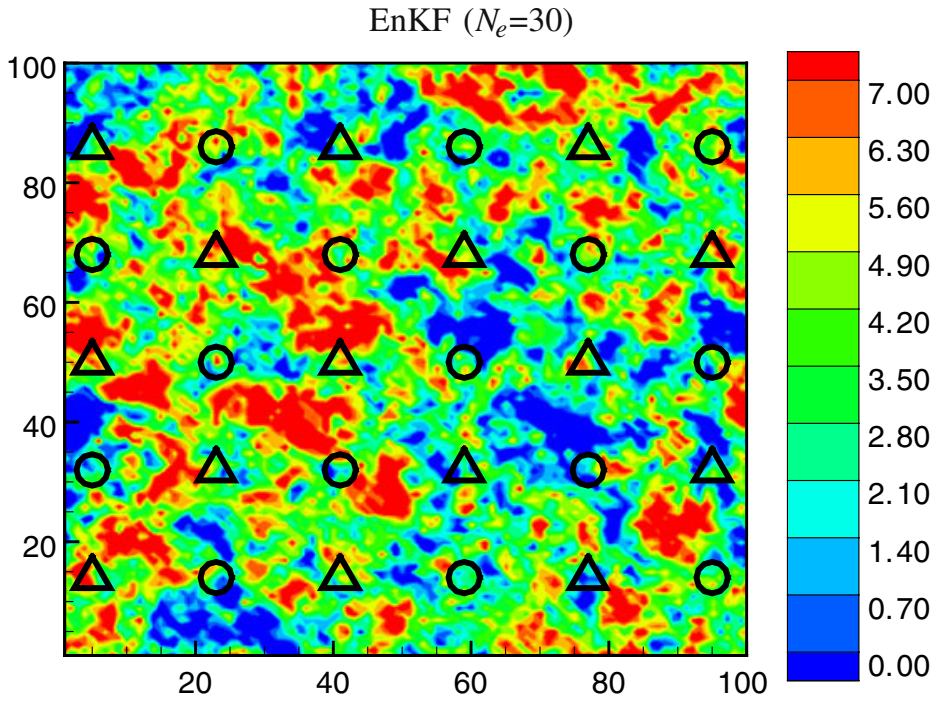

EnKF-LKe

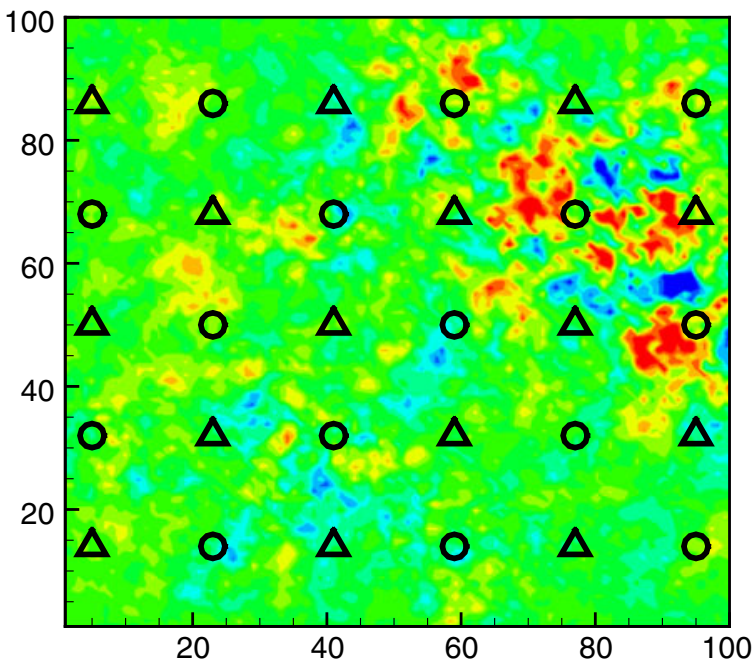

\section{EnKF-SKe}

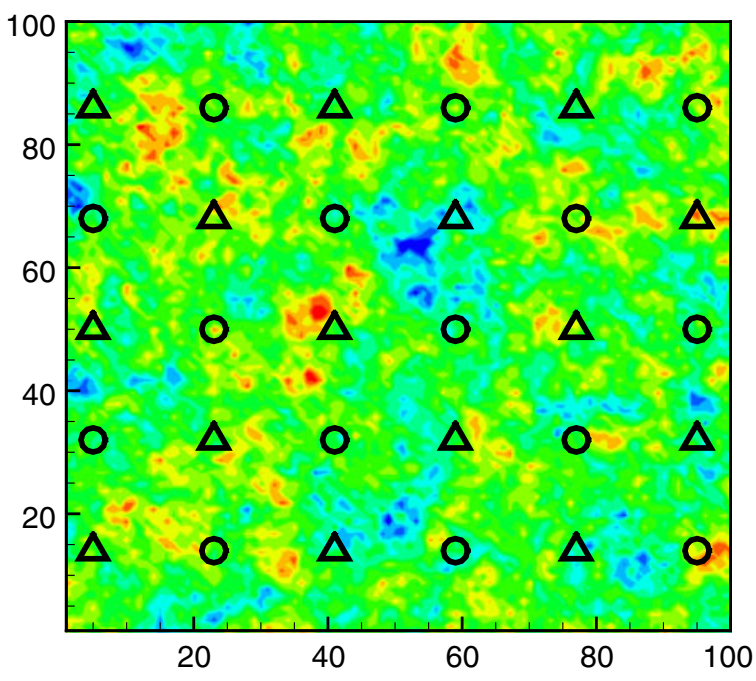

EnKF-LCov

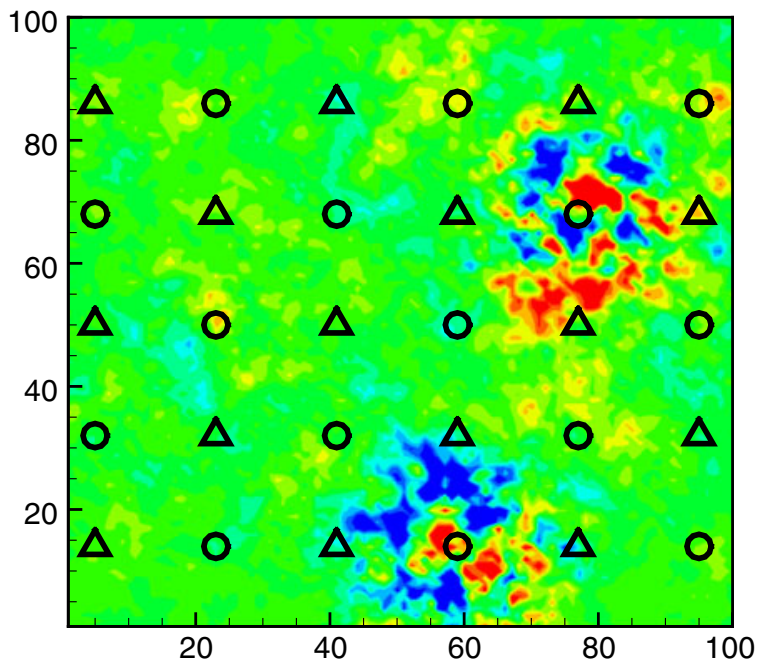

Fig. 17 Final estimates of $\log$ permeability for the example with fault transmissibility multipliers

this section, faults with unknown transmissibilities are incorporated into the reservoir model that was used in the previous example. The objective of this test is to see how the presence of spatially uncorrelated parameters in the state vector affects the localization and screening algorithms involved in the EnKF process. The EnKFSCov method is not evaluated here because of its poor performance in the previous example.

All test settings are the same as those used in the previous example, except that 10 faults are incorpo- rated in the reservoir model as shown in Fig. 15 and that the fault transmissibility multipliers for these 10 faults are to be estimated along with the log permeability at 10,000 gridblocks. The fault geometry is kept simple (rectangular) and all the gridblocks contained in one fault body are assumed to have the same fault transmissibility multiplier. The initial ensemble of the transmissibility multipliers of 10 faults is generated from a uniform distribution between 0.0 and 0.1 .

Table 5 Statistical quantities of the final estimates of log permeability for the example with fault transmissibility multipliers

\begin{tabular}{lllll}
\hline & EnKF $\left(N_{e}=30\right)$ & EnKF-SKe & EnKF-LKe & EnKF-LCov \\
\hline Ensemble STD & 0.006 & 0.15 & 0.97 & 0.70 \\
RMSE & 2.29 & 1.21 & 1.56 & 1.40 \\
\hline
\end{tabular}


Table 6 The average error versus average spread $\left(\hat{e}_{d} / \hat{\sigma}_{d}\right)$ of the predictions of three types of data for the example with fault transmissibility multipliers

\begin{tabular}{llll}
\hline & OPR & WPR & BHP \\
\hline EnKF $\left(N_{e}=30\right)$ & $39 / 0.6$ & $267 / 0.6$ & $5 / 0.8$ \\
EnKF-SKe & $24 / 19$ & $112 / 15$ & $4 / 46$ \\
EnKF-LKe & $28 / 47$ & $292 / 36$ & $3 / 75$ \\
EnKF-LCov & $28 / 35$ & $184 / 26$ & $2 / 51$ \\
\hline
\end{tabular}

At each data assimilation time, truncation was used to maintain the updated fault transmissibility multiplier values within the range of 0.0 and 1.0. The final updated fault transmissibility multipliers obtained at the end of the data assimilation process are shown in Fig. 16. The ensemble estimates of fault transmissibility multipliers from the standard EnKF $\left(N_{e}=30\right)$ have collapsed to values that are quite far from the reference values. The values of some estimates are substantially larger than 0.1. The estimates of fault transmissibility multipliers from EnKF-LKe are similar to those obtained from the standard EnKF $\left(N_{e}=30\right)$ because Kalman gain localization cannot be applied on fault transmissibility multipliers. The EnKF with covariance localization (EnKF-LCov) shows the worst estimates of fault transmissibility multipliers. The localization applied on $C_{d d}^{f}$ seems to have a negative influence on the updating of fault transmissibility multipliers. The EnKF-SKe method provides the best estimates of fault transmissibility multipliers, although the estimates of multipliers for faults 6 and 10 are poor.

The estimates of log permeability from EnKF-LKe and EnKF-LCov appear to have some extreme values (Fig. 17), and EnKF-LCov shows very strong artifacts of the localization. The localization coefficients for EnKF-LCov are based on the distance-dependence assumption which may not be valid in the presence of flow barriers in the reservoir model. Table 5 shows that EnKF-LCov and EnKF-LKe result in larger RMSE values compared to the EnKF-SKe method. The ensemble STD from EnKF-SKe shows the same value as obtained from the previous example, while the ensemble STD from EnKF-LCov and EnKF-LKe vary slightly from their values obtained in the previous example.

With the final updated log permeability fields and fault transmissibility multipliers, we rerun the simula- tions from time 0 to the end of the production period (day 520). Table 6 shows the error and spread of the data predictions from different methods. The EnKFSKe results in the lowest $\hat{e}_{d}$ for OPR and WPR data but shows slightly higher values for BHP data.

Compared to the standard EnKF of the same ensemble size, the EnKF with screening or localization introduces extra computational cost for calculating the Kalman gain of bootstrapped ensembles and for computing the Schur product of the Kalman gain and screening/localizing factors. The extra computational cost of resampling, however, does not necessarily slow down the data assimilation process. On the contrary, Table 7 shows that EnKF with screening Kalman gain (EnKF-SKe) required only about $65 \%$ of the total CPU time needed for the standard EnKF without screening or localization. The reduction in computational time in case of EnKF-SKe can be attributed to improved updates to model parameters leading to faster convergence of the Newton or linear iterations for solving the system equations inside the reservoir simulator.

\section{Conclusions}

In this work, we evaluated and compared several methods of regularizing the Kalman gain and regularizing the covariance matrices used for computation of the Kalman gain. The performance of the methods was based on improvement in the estimates of the Kalman gain, quality of data prediction, and the estimates of model variables. Distance-dependent localization and bootstrap-based screening were both evaluated. The error analysis carried out in the 1D linear example showed that estimation of the Kalman gain by covariance regularization is more error prone than direct regularization of the Kalman gain regularization. This point is clearly illustrated by the dramatically different performance of Kalman gain screening and covariance screening. The performances of the distance-based covariance localization and Kalman gain localization, however, are not significantly different when the state vector contains only spatially correlated variables. This is probably because knowledge of the correlation length, sensitivity, and well pattern used for construct-
Table 7 CPU time (minutes) for the example with fault transmissibility multipliers using three processors

\begin{tabular}{llccc}
\hline & EnKF $\left(N_{e}=30\right)$ & EnKF-SKe & EnKF-LKe & EnKF-LCov \\
\hline Forward time & 10.5 & 9 & 7.4 & 6.4 \\
Assimilation time & 1.5 & 3 & 1.6 & 1.6 \\
Rerun time & 12 & 4 & 6 & 4 \\
Total CPU time & 24 & 16 & 15 & 12 \\
\hline
\end{tabular}


ing the localization coefficients substantially reduces the error in the coefficients.

We also showed that when regularization is applied to the covariance matrices, a consistency condition must be satisfied. For the problem of assimilating multiple, non-local observations, it is difficult to satisfy the consistency condition for the distance-dependent covariance localization. In the 2D nonlinear example, an approximately consistent form of covariance localization was applied with acceptable results in terms of matching data and maintaining ensemble effective rank. Some extreme values were observed in the final estimates of log permeability fields, however, especially for the case of estimating fault transmissibility multipliers. Certainly, these extreme values are not only caused by inconsistency but also the assumption that the true correlations can be localized spatially. One key limitation of distance-based localization is that, when the distance from a gridblock to the data location is beyond the specified range, the correlation value at that gridblock is assumed to be zero, in which case, the Kalman gain value at that gridblock is determined only by the data whose correlation at that gridblock is nonzero. This can result in magnification of the influence from a particular data, which leads to over-correction on model variables. The distance-dependence assumption appears to be delicate and should probably be used with caution in the presence of complex geology.

The results from both 1D and 2D examples clearly show that screening Kalman gain (EnKF-SKe) worked well on a variety of problems with few assumptions. In the algorithm of screening Kalman gain, we directly calculate the replicate of Kalman gain from each bootstrap ensemble and quantify the confidence level of the Kalman gain directly from the $N_{B}$ bootstrap replicates of the Kalman gain. No assumption about the prior covariance is required in this case. The method can be used for estimating both spatially correlated and uncorrelated variables. Despite the apparent cost of resampling the Kalman gain multiple times, the total computational cost for EnKF-SKe was less than that for standard EnKF because of reduced time for reservoir simulation.

Acknowledgements The first author acknowledges financial support from the OU Consortium on Ensemble Methods. The authors would like to acknowledge the donation of multiple licenses of ECLIPSE by Schlumberger and computational resources provided by the OU Supercomputing Center for Education and Research.

Open Access This article is distributed under the terms of the Creative Commons Attribution Noncommercial License which permits any noncommercial use, distribution, and reproduction in any medium, provided the original author(s) and source are credited.

\section{References}

1. Agbalaka, C.C., Oliver, D.S.: Application of the EnKF and localization to automatic history matching of facies distribution and production data. Math. Geosci. 40(4), 353-374 (2008)

2. Anderson, J.L.: Exploring the need for localization in ensemble data assimilation using a hierarchical ensemble filter. Phys. Nonlinear Phenom. 230(1-2), 99-111 (2007)

3. Bergemann, K., Reich, S.: A localization technique for ensemble Kalman filters. Q. J. R. Meteorol. Soc. 136(648, Part A), 701-707 (2010). doi:10.1002/qj.591

4. Chen, Y., Oliver, D.: Cross-covariances and localization for EnKF in multiphase flow data assimilation. Comput. Geosci. 14, 579-601 (2010). doi:10.1007/s10596-009-9174-6

5. Chen, Y., Oliver, D.S.: Ensemble-based closed-loop optimization applied to Brugge Field. SPE Reserv. Evalu. Eng. 13(1), 56-71 (2010). doi:10.2118/118926-PA

6. Efron, B., Tibshirani, R.J.: An Introduction to the Bootstrap, 1st edn. Chapman \& Hall/CRC, Boca Raton (1993)

7. Evensen, G.: Sequential data assimilation with a nonlinear quasi-geostrophic model using Monte Carlo methods to forecast error statistics. J. Geophys. Res. 99(C5), 10, 143-10, 162 (1994)

8. Evensen, G., Hove, J., Meisingset, H.C., Reiso, E., Seim, K.S., Espelid, $\varnothing .:$ Using the EnKF for assisted history matching of a North Sea reservoir model (SPE 106184). In: Proceedings of the 2007 SPE Reservoir Simulation Symposium (2007)

9. Fertig, E.J., Hunt, B.R., Ott, E., Szunyogh, I.: Assimilating non-local observations with a local ensemble Kalman filter. Tellus A 59(5), 719-730 (2007). doi:10.1111/j.16000870.2007.00260.x

10. Furrer, R., Bengtsson, T.: Estimation of high-dimensional prior and posterior covariance matrices in Kalman filter variants. J. Multivar. Anal. 98(2), 227-255 (2007). doi:10.1016/ j.jmva.2006.08.003

11. Gu, Y., Oliver, D.S.: History matching of the PUNQ-S3 reservoir model using the ensemble Kalman filter. SPE J. 10(2), 51-65 (2005). doi:10.2118/89942-PA

12. Hamill, T.M., Whitaker, J.S., Snyder, C.: Distance-dependent filtering of background error covariance estimates in an ensemble Kalman filter. Mon. Weather Rev. 129(11), 2776-2790 (2001)

13. Haugen, V., Naevdal, G., Natvik, L.J., Evensen, G., Berg, A.M., Flornes, K.M.: History matching using the ensemble Kalman filter on a North Sea field case. SPE J. 13(4), 382391 (2008)

14. Houtekamer, P.L., Mitchell, H.L.: Data assimilation using an ensemble Kalman filter technique. Mon. Weather Rev. 126(3), 796-811 (1998)

15. Houtekamer, P.L., Mitchell, H.L.: A sequential ensemble Kalman filter for atmospheric data assimilation. Mon. Weather Rev. 129(1), 123-137 (2001)

16. Houtekamer, P.L., Mitchell, H.L., Pellerin, G., Buehner, M., Charron, M., Spacek, L., Hansen, B.: Atmospheric data assimilation with an ensemble Kalman filter: results with real observations. Mon. Weather Rev. 133(3), 604-620 (2005)

17. Lorenc, A.C.: The potential of the ensemble Kalman filter for NWP - a comparison with 4D-Var. Q. J. R. Meteorol. Soc. 129(595), 3183-3203 (2003) 
18. Nævdal, G., Johnsen, L.M., Aanonsen, S.I., Vefring, E.H.: Reservoir monitoring and continuous model updating using ensemble Kalman filter. SPE J. 10(1), 66-74 (2005). doi:10.2118/84372-PA

19. Schlumberger (2006) Eclipse Technical and Reference Manual. Schlumberger, Abingdon
20. Zhang, Y., Oliver, D.S.: History matching using a multiscale stochastic model with the ensemble Kalman filter: a field case study. SPE J. (2010). doi:10.2118/118879-PA

21. Zhang, Y., Oliver, D.S.: Improving the ensemble estimate of the Kalman gain by bootstrap sampling. Math. Geosci. 42(3), 327-345 (2010). doi:10.1007/s11004-010-9267-8 